\title{
Resonance fluorescence in photonic band gap waveguide architectures: Engineering the vacuum for all-optical switching
}

\author{
Marian Florescu* and Sajeev John \\ Department of Physics, University of Toronto, Toronto, Ontario, Canada M5S 1A7
}

(Received 22 December 2003; published 11 May 2004)

\begin{abstract}
We describe the spectral characteristics of the radiation scattered by two-level atoms (quantum dots) driven by a strong external field, and coupled to a photonic crystal radiation reservoir. We show that in the presence of strong variations with the frequency of the photonic reservoir density of states, the atomic, Mollow, sideband components of the scattered intensity can be strongly modified. Consequently, a weak optical probe field experiences a substantial differential gain in response to slight variations in the intensity of an optical driving field. We suggest that these effects may be of relevance to all-optical transistor action in photonic crystals. Using a specific photonic crystal heterostructure, we suggest that an all-optical microtransistor based on photonic crystals may operate at less than $100 \mathrm{nW}$ switching threshold power. Collective $N$-atom effects substantially enhance this optical switching effect. Near the switching threshold intensity, collective effects are manifest in the $N^{2}$ scaling of the intensity spectrum (reminiscent of superradiance). Above and below this critical region, the gain spectrum widens (linearly with $N$ ). This correspondingly reduces the switching time scales of the atomic system in response to external fields. Furthermore, the quantum degree of second-order coherence exhibits unusual features. Scattered photons display a variable degree of antibunching as function of driving laser field intensity and the photonic density of states discontinuity. We analyze the effects of the inhomogeneous atomic line broadening on the amplification process. We show, using suitable photonic density of states engineering, that it is possible to select a narrow spectral range around the central frequency of the atomic frequency distribution over which amplification and switching occur. This is done either by spectral decoupling of the active elements from the electromagnetic field (through the introduction of band gaps at specified spectral locations) or through incoherent pumping to selectively saturate atoms outside the spectral region chosen for amplification.
\end{abstract}

DOI: 10.1103/PhysRevA.69.053810

PACS number(s): 42.50.Ct, 42.50.Hz, 32.80.- $\mathrm{t}$

\section{INTRODUCTION}

Photonic band gap (PBG) material crystals constitute a class of periodically ordered dielectrics that carry the concept of moulding and controlling the flow of light to its most microscopic level. This is entailed in a fundamentally optical principle, namely the localization of light. The synergetic interplay between the microcavity resonances of individual dielectric unit cells and the Bragg scattering resonances of the overall dielectric array [1] controllably alters the basic electromagnetic interaction over certain frequency and length scales. This leads to a range of frequencies (PBG) for which no propagating electromagnetic modes are allowed $[2,3]$. The presence of the photonic band gap in the dispersion relation of the electromagnetic field gives rise to phenomena, such as the inhibition of the spontaneous emission [4], strong localization of light [5], formation of atom-photon bound states [6], single-atom and collective atomic switching behavior by coherent resonant pumping, and atomic inversion without fluctuations [7-9].

In this paper, we present a detailed analysis of the dynamics and the spectral properties of the radiation emitted by a

\footnotetext{
*Present address: Jet Propulsion Laboratory, California Institute of Technology, Mail Stop 126-347, 4800 Oak Grove Drive, Pasadena, CA 91109, USA.

Electronic address: marian@physics.utoronto.ca
}

single atom or a collection of $N$ atoms, coherently driven by an external laser field and coupled to the radiation reservoir of a photonic crystal. The phenomenon of resonance fluorescence has attracted considerable experimental interest over the years and has been a test bed for the development of quantum theories of matter-radiation interaction. Of particular interest is the highly nonlinear character of this phenomenon. If the intensity of the driving field is sufficiently high, the atom laser field interaction is dominated by multiphoton photon scattering processes. The presence of the frequencydependent radiation reservoir of a photonic crystal provides an opportunity to engineer the resonance fluorescence process. We show that the competition between the drivingfield-induced nonlinearities and the feedback provided by the photonic crystal gives rise to phenomena in the response of the atomic system to the external excitation. In particular, we demonstrate that the character of the absorption spectrum of a probe (signal) field can be switched by small variations in intensity of the driving (control) field.

A suitably doped PBG material may, in the sense described above, exhibit ultrafast all-optical switching and alloptical transistor action. Similar to other all-optical transistor proposals [10], the transition between the absorptive and the amplifying regime can be modulated with a weak second control laser field (in phase and in resonance with the main strong pump field). Using a specific architecture (presented in Fig. 1), involving two waveguide modes within a photonic band gap, we suggest that (in contrast to earlier proposals) 


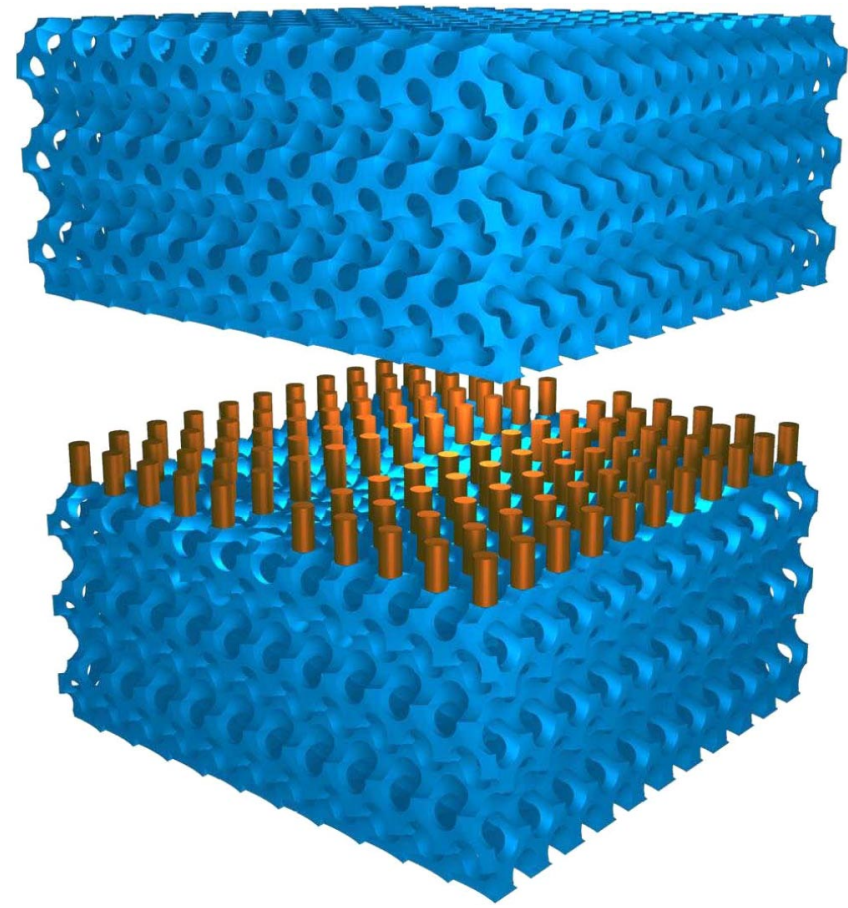

FIG. 1. Photonic band gap waveguide architecture for all-optical switching and transistor action. The microstructure consists in a waveguide channel in a two-dimensional photonic crystal, which is embedded in a 3D photonic crystal [11].

switching is not limited by the conventional (inverse) relationship between the switching (cavity buildup) time and the switching intensity threshold [10]. Instead of a narrow cavity resonance, the PBG based switching effect requires the coupling of light emitters to a pair of electromagnetic waveguide modes. As schematically presented in Fig. 2, one waveguide exhibits a sharp cutoff over a narrow frequency interval within the three dimensional (3D) PBG. The low group velocity of this mode is suitable for conveying a steady-state holding field to the atomic system. Another waveguide mode with high group velocity in the frequency range of the atomic resonance is suitable for coupling light from a modulation (control) field and a probe beam to the same atomic system. As we show, a probe laser field of prescribed wavelength will experience a substantial differential gain by slight modulations in the weak control laser field. The high group velocity of the second waveguide mode facilitates fast switching without compromising the low switching threshold provided by the sharp cutoff of the first waveguide mode.

The ability of an external pump laser to switch the spectral characteristics of the atomic system across the band edge [or near any other discontinuous density of states DOS profile] leads to coherent all-optical switching and transistor action. In order to estimate the intensities required to observe all-optical switching and transistor action in photonic crystals, it is useful to introduce the concept of "quality factor of a band edge." Analogous to the quality $Q$ factor of a conventional microcavity, we define the quality factor of a band edge as the ratio between the central frequency and the frequency range over which the photonic DOS presents a predetermined variation (see Fig. 3). Preliminary calculations

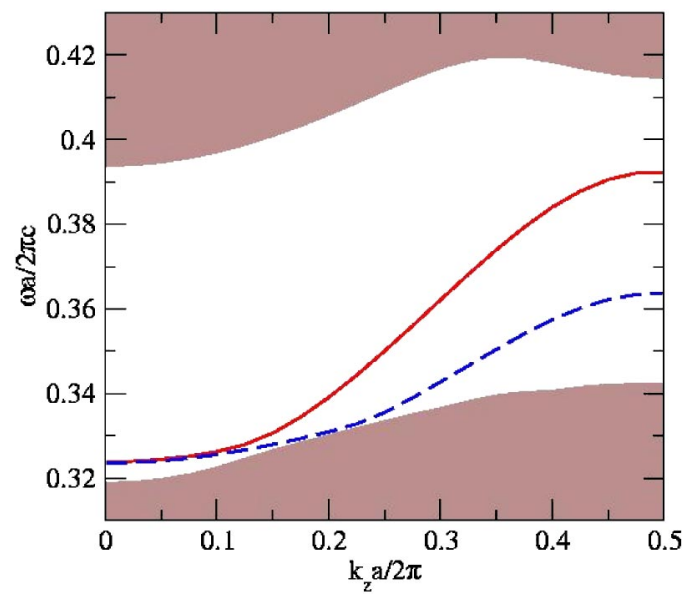

FIG. 2. Dispersion relation of a photonic crystal heterostructure similar to one presented in Fig. 1 for propagation along the waveguide. The two-dimensional photonic crystal consists of Si cylinders with aspect ratio $r / a=0.3$ (here $r$ is cylinder radius and $a$ dielectric lattice constant), and cylinder height of $h=0.4 a$. The linear waveguide is generated by removing three rows of cylinders in the longitudinal direction. The three-dimensional photonic crystal is assumed to be an inverted square spiral structure (Si infiltrated) that presents a photonic band gap of 23.6\% [13]. For these parameters, the linear defect supports two waveguide modes, one of which experiences a sharp cutoff in the middle of the 3D PBG, same spectral range for which the second guided mode has a very large dispersion. Source: Ref. [12].

[12] show that in a microstructure consisting of a waveguide channel in a 3D PBG heterostructure, it is possible to achieve very large band-edge quality factors. For example, for a sample of 20 unit cells in length and a jump in local density of states (LDOS) of a factor of 100, the band-edge quality factor is about $10^{5}$, and, by increasing the length of the structure, the quality factor may be further increased (i.e., the density of states changes by a factor of 100 on a spectral range of $1-10 \mathrm{GHz}$ ). In this case, (for frequencies in the optical domain) a $1 \mu \mathrm{W}$ continuous-wave laser field guided through a waveguide channel of cross section $1 \times 1 \mu \mathrm{m}^{2}$ produces an intensity of $0.1 \mathrm{~kW} / \mathrm{cm}^{2}$ and will generate the holding intensity required to observe all-optical transistor action (the Rabi frequency associated with this driving field is in the 10-20 GHz range). For such small areas, it is likely that the most efficient way of coherently driving the active elements may be using a microlaser [14] built into the photonic crystal. Moreover, in a photonic crystal, due to field focusing effects, it is possible to achieve very large localfield enhancement (at certain positions inside the photonic crystal, the intensity of specific electromagnetic modes can be increased from its empty space value by factors as large as 600 [15]). These effects may reduce the holding power required to observe transistor action on the $10-\mathrm{nW}$ scale, while the switching power (intensity modulation) may be as low as $500 \mathrm{pW}$.

We also analyze the higher-order optical correlation functions as they are modified by resonance fluorescence near the waveguide cutoff in the photonic crystal. A comparison between our model results and experimentally measured degree 


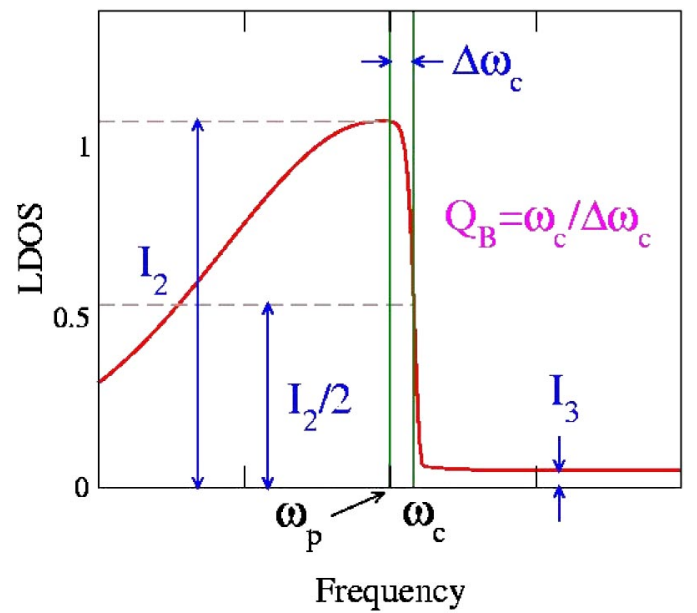

FIG. 3. A schematic description of the local density of states (LDOS) generated by the structure presented in Figs. 1 and 2, which illustrates the concept of band-edge quality factor. The spectral range used here roughly corresponds to the middle of the gap in Fig. 2, and the large dispersion mode (continuous curve in Fig. 2) generates a relatively small contribution to the LDOS, while the sharp frequency cutoff of the second guided mode (the dashed curve in Fig. 2) gives rise to the apparent jump in the LDOS. The band-edge quality factor is defined (analogously to the quality factor of a cavity) by the ratio between the central frequency $\omega_{C}$ and the frequency $\Delta \omega_{C}$ range over which the LDOS jumps to half of its peak, i.e., $Q_{B}=\omega_{C} / \Delta \omega_{C}$.

of second-order coherence could be used in extracting the magnitude of the jump of the photonic DOS and the amount of dipolar dephasing present in the system. When the system consists of a single atom (or a collection of $N$ identical atoms) exhibiting only natural linewidth, the gain spectrum of the probe beam is relatively narrow. The important effects associated with the inhomogeneous line broadening of the atomic (or quantum dot) ensemble are investigated as well. We show that for various inhomogeneously broadened ensembles, the gain spectrum may be either broadened or washed out, depending on the details of the pump field and the details of the engineered vacuum density of states.

\section{DYNAMICS OF ATOMIC VARIABLES}

Consider a two-level atomic system interacting with a radiation field reservoir of a confined photonic crystal, and driven by a "strong" ( $10 \mathrm{nW}-1 \mu \mathrm{W})$ classical laser field [8]. The atomic system is characterized by the states $|1\rangle$ (ground state) $|2\rangle$ (excited state), the resonant atomic transition $\omega_{A}$, and may interact with the lattice vibrations of the dielectric host material. In a rotating wave approximation basis [7-9] corresponding to the laser frequency $\omega_{L}$ and in the interaction picture, the Hamiltonian of the system has the form

$$
H=H_{0}+H_{A F}+H_{A L}+H_{\text {deph }} .
$$

Here $H_{0}=H_{A}+H_{R}$ characterizes the free evolution of the atomic system and photonic reservoir in the absence of atomradiation interaction, $H_{A F}$ represents the coupling between the atomic system and the radiation reservoir of the photonic crystal, and $H_{\text {deph }}$ describes additional dephasing effects that may arise from atomic collisions or the scattering of phonons from quantum dots embedded in a solid part of the dielectric structure. The noninteracting system is described by

$$
H_{0}=\hbar \Delta_{A L} \sigma_{3}+\sum_{\lambda} \hbar \Delta_{\lambda} a_{\lambda}^{\dagger} a_{\lambda}
$$

the interaction of the atomic collection with the electromagnetic modes of the photonic reservoir is given by

$$
H_{A F}=-i \hbar \sum_{\lambda} g_{\lambda}\left(a_{\lambda}^{\dagger} \sigma_{-}-\sigma_{+} a_{\lambda}\right)
$$

and the interaction of the atomic system with the external laser field has the form

$$
H_{A L}=\hbar \epsilon\left(\sigma_{-}-\sigma_{+}\right) .
$$

Here, $\sigma_{-}, \sigma_{+}$, and $\sigma_{3}$ describe the excitation, the deexcitation, and the atomic inversion operators of the atomic system, respectively [7-9]; $a_{\lambda}, a_{\lambda}^{\dagger}$ are the radiation annihilation operators for photons of the allowed modes $(\lambda$ $\equiv\left\{\mathbf{k}_{\lambda}, \mathbf{e}_{\lambda}\right\}$, where $\mathbf{k}_{\lambda}$ is the wave vector of the radiation mode $\lambda, \mathbf{e}_{\lambda}$ are the two transverse unit polarization directions). $\Delta_{\lambda}=\omega_{\lambda}-\omega_{L}$ and $\Delta_{A L}=\omega_{A}-\omega_{L}$ are $\lambda$-mode frequency detuning and the atom frequency detuning with respect to the laser field frequency, $\omega_{L}$, respectively. $g_{\lambda}$ is the coupling constant between the atom and the reservoir $\lambda$ mode,

$$
g_{\lambda}=\frac{\omega_{A} \mu}{\hbar}\left(\frac{\hbar}{2 \varepsilon_{0} \omega_{\lambda} \mathcal{V}}\right)^{1 / 2} \mathbf{e}_{\lambda} \cdot \mathbf{u}
$$

where $\mathbf{u}$ and $\mu$ are the unit vector and the absolute value of the dipole moment of the atom. Also, $\mathcal{V}$ is the volume of the sample and $\varepsilon_{0}$ is the Coulomb constant. Finally, $\boldsymbol{\epsilon}=\boldsymbol{\mu} \cdot \mathbf{E} / \hbar$ is the resonant Rabi frequency of the atom and $\mathbf{E}$ is the applied laser field amplitude. The Hamiltonian (2.1) presents two types of radiation field-matter interaction: a coherent interaction $\left(H_{A L}\right)$ between the external applied field and the atomic system, and an incoherent interaction $\left(H_{A F}\right)$ between the atom and the electromagnetic modes of the photonic crystal reservoir. The latter describes the dissipation of atomic deexcitation energy into the radiation field (consisting of a "bath" of quantum fluctuations of the photonic crystal vacuum).

The analysis is simplified under the assumption that the photonic mode density, while discontinuous at specific frequencies, is constant over the spectral regions surrounding the dressed resonant frequencies $\omega_{L}, \omega_{L}-2 \Omega$, and $\omega_{L}+2 \Omega$ (where $\Omega=\left[\epsilon^{2}+\Delta_{A L}^{2} / 4\right]^{1 / 2}$ is the generalized Rabi frequency), as shown in Fig. 4. However, as a result of the frequencydependent character of the photonic reservoir DOS, is is not possible to write a master equation in the bare atomic picture. Consequently, we introduce an atomic basis change from the bare atomic states $\{|1\rangle,|2\rangle\}$ to the dressed atomic states $\{|\tilde{1}\rangle,|\widetilde{2}\rangle\}$, defined by 


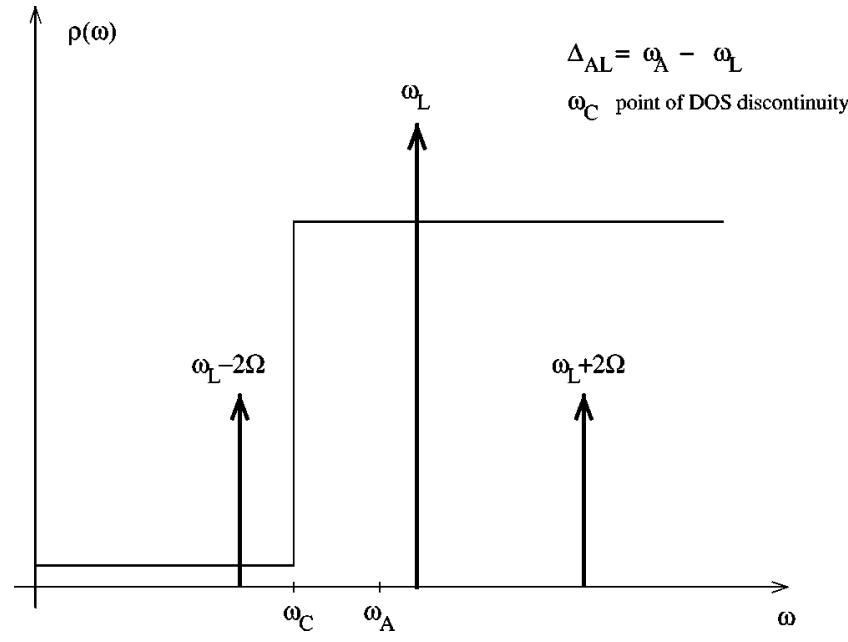

FIG. 4. Relevant frequencies and frequency scales in the case of a steplike density of states.

$$
\begin{gathered}
|\widetilde{1}\rangle=c|1\rangle+s|1\rangle, \\
|\widetilde{2}\rangle=-s|1\rangle+c|1\rangle .
\end{gathered}
$$

The corresponding dressed atomic operators $R_{ \pm}, R_{3}$ are related to the bare atomic operators by the relations

$$
\begin{gathered}
\sigma_{-}=c s R_{3}+c^{2} R_{-}-s^{2} R_{+}, \\
\sigma_{+}=c s R_{3}-s^{2} R_{-}+c^{2} R_{+}, \\
\sigma_{3}=\left(c^{2}-s^{2}\right) R_{3}-2 c s\left(R_{-}+R_{+}\right),
\end{gathered}
$$

with $s$ and $c$ defined by

$$
\begin{aligned}
& \sin ^{2}(\phi) \equiv s^{2}=\frac{1}{2}\left[1-\frac{1}{2} \frac{\Delta_{A L}}{\Omega}\right], \\
& \cos ^{2}(\phi) \equiv c^{2}=\frac{1}{2}\left[1+\frac{1}{2} \frac{\Delta_{A L}}{\Omega}\right] .
\end{aligned}
$$

In the dressed basis, the total Hamiltonian is now given by $H=H_{0}+H_{I}$, with

$$
\begin{gathered}
H_{0}=\sum_{\lambda} \hbar \Delta_{\lambda} a_{\lambda}^{\dagger} a_{\lambda}+\hbar \Omega R_{3}, \\
H_{I}=i \hbar g_{\lambda}\left[a_{\lambda}^{\dagger}\left(c s R_{3}+c^{2} R_{-}-s^{2} R_{+}\right)\right]+\text {H.c. }
\end{gathered}
$$

Furthermore, we introduce the time-dependent interaction picture generated by the unitary operator $\exp \left(-i H_{0} t\right)$, such that the interaction Hamiltonian becomes

$$
\begin{aligned}
\widetilde{H}_{I}(t)= & i \hbar g_{\lambda}\left[a_{\lambda}^{\dagger}\left(c s R_{3} e^{i \Delta_{\lambda} t}+c^{2} R_{-} e^{i\left(\Delta_{\lambda}-2 \Omega\right) t}-s^{2} R_{+} e^{i\left(\Delta_{\lambda}+2 \Omega\right) t}\right)\right] \\
& + \text { H.c. }
\end{aligned}
$$

The dressed atomic operators in this interaction picture exhibit the time dependence given by $\widetilde{R}_{-}(t)=R_{-}(0) e^{-2 i \Omega t}$, $\widetilde{R}_{+}(t)=R_{+}(0) e^{2 i \Omega t}$, and $\widetilde{R}_{z}(t)=R_{3}(0)$. Hereafter, we drop the tilde on the interaction picture operators. Formally, the influence of the driving field on the atom-radiation interaction is embedded in the memory functions

$$
\begin{gathered}
G_{0}\left(t-t^{\prime}\right)=\sum_{\lambda} g_{\lambda}^{2} e^{-i \Delta_{\lambda}\left(t-t^{\prime}\right)}, \\
G_{ \pm}\left(t-t^{\prime}\right)=\sum_{\lambda} g_{\lambda}^{2} e^{-i\left(\Delta_{\lambda} \mp 2 \Omega\right)\left(t-t^{\prime}\right)},
\end{gathered}
$$

which, in turn, are determined by the radiation field density of states. For a broadband, smoothly varying density of states (as in ordinary vacuum), the dependence of the memory functions on the external field can be ignored and $G_{0}\left(t-t^{\prime}\right)$ $=G_{ \pm}\left(t-t^{\prime}\right) \approx(\gamma / 2) \delta\left(t-t^{\prime}\right)$. However, the density of states of the photonic crystals exhibits band-edge and other Van Hove singularities. In systems with fast variations of the density of states in the spectral range given by $\left\{\omega_{L}-2 \Omega, \omega_{L}+2 \Omega\right\}$ (shown in Fig. 4), the distinctive memory functions introduced in Eq. (2.11) lead to qualitatively different behavior from ordinary vacuum.

We consider that the atomic system is driven by a "strong" $(10 \mathrm{nW}-1 \mu \mathrm{W})$ (focused into a submicron waveguide channel) [7], such that the dressed frequencies $\omega_{L}$, $\omega_{L}-2 \Omega$, and $\omega_{L}+2 \Omega$ are pushed away from the DOS discontinuities, even though the original atomic transition may coincide with a discontinuity. The "strong-field" assumption simplifies the analysis by ensuring that the Mollow spectral components are well separated and the overlap between them is negligible. In this case, the spectral components of the scattered spectrum will experience very different densities of states and the memory functions in the Markov approximation are given by $G_{0}\left(t-t^{\prime}\right)=\left(\gamma_{0} / 2\right) \delta\left(t-t^{\prime}\right), G_{ \pm}\left(t-t^{\prime}\right)$ $=\left(\gamma_{ \pm} / 2\right) \delta\left(t-t^{\prime}\right)$ [8]. The spontaneous emission decay rates $\gamma_{0}=2 \pi \Sigma_{\lambda} g_{\lambda}^{2} \delta\left(\omega_{\lambda}-\omega_{L}\right), \gamma_{ \pm}=2 \pi \Sigma_{\lambda} g_{\lambda}^{2} \delta\left(\omega_{\lambda}-\omega_{L} \mp 2 \Omega\right)$ are proportional to the density of modes at dressed-state resonant frequencies.

Under these conditions, the usual formalism of open systems [16] can be employed to obtain the reduced master equation describing the evolution of the atomic system. In order to obtain a more realistic picture, we assume that, in addition to the radiative coupling of the atomic system to the electromagnetic reservoir of the photonic crystal, the atomic system interacts with a nonradiative reservoir that describes additional dephasing interactions. These may arise from scattering of phonons (if quantum dots are embedded in the solid part of a photonic crystal) or from interatomic collisions (if "cold" atoms are trapped in the voids of the dielectric structure). The dephasing is characterized by a phenomenological decay rate $\gamma_{p}=1 / T_{2}^{n r}$. We assume that the radiative and the dephasing reservoirs are statistically independent, such that we can evaluate separately their contributions to the master equation describing the temporal evolution of the atomic system. We also consider the interaction between the atomic system and the reservoirs to be turned on at $t=0$ (i.e., initially, there are no correlations between the system and the reservoir) and make the Born approximation [16] (in which we discard terms containing contribution higher than second order in the atomic system-reservoir interaction). In the in- 
teraction picture, the evolution of the atomic system is described by the following reduced master equation [16]:

$$
\left[\frac{\partial \widetilde{\rho}(t)}{\partial t}\right]_{\text {rad }}=-\frac{1}{\hbar^{2}} \int_{0}^{t} d t^{\prime} t r_{R}\left\{\left[\widetilde{H}_{S R}(t),\left[\widetilde{H}_{S R}\left(t^{\prime}\right), \widetilde{\rho}\left(t^{\prime}\right) R_{0}\right]\right]\right\} .
$$

Here, $\widetilde{\rho}(t)$ is the reduced density operator of the atomic system (obtained after summation over the reservoir degrees of freedom has been performed). $H_{S R}(t)$ represents a generic interaction Hamiltonian between the atomic system and the reservoir [in our analysis, $H_{S R}$ is given by Eq. (2.10)]. $R_{0}$ is the initial density operator of the photonic reservoir (the reservoir state is assumed to be virtually unchanged by the its coupling to the atomic system), which, for simplicity, is considered to be the vacuum state. The modal density of states depicted in Fig. 4 allows us to make a simplifying Markov approximation, in which we replace $\tilde{\rho}\left(t^{\prime}\right)$ by $\tilde{\rho}(t)$ in Eq. (2.12).

The reduced master equation for the atomic system in the dressed atomic basis is given by [17]

$$
\frac{d \rho(t)}{d t}=\left.\frac{d \rho(t)}{d t}\right|_{\text {sec }}+\left.\frac{d \rho(t)}{d t}\right|_{ \pm 2 \Omega}+\left.\frac{d \rho(t)}{d t}\right|_{ \pm 4 \Omega},
$$

where

$$
\begin{aligned}
&\left.2 \frac{d \rho(t)}{d t}\right|_{s e c}=-A_{0}\left[\rho(t)-R_{3} \rho(t) R_{3}\right]-A_{-}\left[R_{11} \rho(t)+\rho(t) R_{11}\right. \\
&\left.-2 R_{21} \rho(t) R_{12}\right]-A_{+}\left[R_{22} \rho(t)+\rho(t) R_{22}\right. \\
&\left.-2 R_{12} \rho(t) R_{21}\right], \\
&\left.2 \frac{d \rho(t)}{d t}\right|_{ \pm 2 \Omega}= c s e^{-2 i \Omega t}\left\{s^{2} \gamma_{0}\left[R_{12} \rho(t)-R_{3} \rho(t) R_{12}\right]\right. \\
&+c^{2} \gamma_{0}\left[R_{12} \rho(t) R_{3}+\rho(t) R_{12}\right]-c^{2} \gamma_{+}\left[R_{3} \rho(t) R_{12}\right. \\
&\left.\left.-\rho(t) R_{12}\right]+s^{2} \gamma_{-}\left[R_{12} \rho(t)-R_{12} \rho(t) R_{3}\right]\right\}+ \text { H.c. }, \\
&\left.2 \frac{d \rho(t)}{d t}\right|_{ \pm 4 \Omega}=c^{2} s^{2} e^{4 i \Omega t}\left(\gamma_{-}+\gamma_{+}\right) R_{12} \rho(t) R_{12}+\text { H.c. },
\end{aligned}
$$

with $A_{0}=\gamma_{0} c^{2} s^{2}+\gamma_{p}\left(c^{2} s^{2}\right)^{2}, \quad A_{-}=\gamma_{-} s^{4}+4 \gamma_{p} c^{2} s^{2}, \quad$ and $A_{+}$ $=\gamma_{+} c^{4}+4 \gamma_{p} c^{2} s^{2}$. In deriving Eq. (2.13), we have assumed that the spontaneous decay rates experienced by the spectral components of the scattered radiation are proportional to the density of modes at the dressed-state transition frequencies $\omega_{L}, \omega_{L} \pm 2 \Omega\left(\gamma_{0}\right.$ and $\gamma_{ \pm}$, respectively), and we have isolated the fast oscillating terms at frequencies $\pm 2 \Omega, \pm 4 \Omega$. Moreover, we have neglected the small energy shifts caused by atom-reservoir interaction.

Using the master equation, Eq. (2.13), the expectation values of atomic operators obey the following equations of motion:

$$
\begin{aligned}
\frac{d}{d t}\left\langle R_{21}(t)\right\rangle= & -\frac{4 A_{0}+A_{-}+A_{+}}{2}\left\langle R_{21}(t)\right\rangle+c s\left[c^{2} \frac{\gamma_{+}}{2}\right. \\
& \left.-s^{2} \frac{\gamma_{-}}{2}\right] e^{-2 i \Omega t}\left\langle R_{3}(t)\right\rangle+c s\left[c^{2} \frac{\gamma_{+}}{2}+s^{2} \frac{\gamma_{-}}{2}\right. \\
& \left.+\gamma_{0}\right] e^{-2 i \Omega t}-c^{2} s^{2}\left[\frac{\gamma_{-}}{2}+\frac{\gamma_{+}}{2}\right] e^{-4 i \Omega t}\left\langle R_{12}(t)\right\rangle,
\end{aligned}
$$

$$
\begin{aligned}
\frac{d}{d t}\left\langle R_{3}(t)\right\rangle= & -\left[A_{-}+A_{+}\right]\left\langle R_{3}(t)\right\rangle+\left[A_{-}+A_{+}\right]+\gamma_{0} c s\left[c^{2}\right. \\
& \left.-s^{2}\right] e^{-2 i \Omega t}\left\langle R_{12}(t)\right\rangle+\gamma_{0} c s\left[c^{2}-s^{2}\right] e^{2 i \Omega t}\left\langle R_{21}(t)\right\rangle .
\end{aligned}
$$

For "strong" external laser fields (as defined above) or large detunings $\left(\epsilon\right.$ or $\left.\Delta_{A L} \gg \gamma_{0, \pm}\right)$, the atoms undergo many stimulated emission and absorption processes before spontaneously emitting a photon. In this case, the terms in Eq. (2.14) that oscillate at the frequencies $\pm 2 \Omega$ and $\pm 4 \Omega$ will average to zero on the characteristic atomic time scale. In the secular approximation, we neglect the fast oscillating terms at frequencies $\pm 2 \Omega, \pm 4 \Omega$. In this approximation, the equations of motion can be solved analytically, and the results are cast in a very simple form:

$$
\begin{gathered}
\left\langle R_{ \pm}(t)\right\rangle=\left\langle R_{ \pm}(0)\right\rangle e^{-\Gamma_{c o h} t}, \\
\left\langle R_{3}(t)\right\rangle=\left[\left\langle R_{3}(0)\right\rangle-\left\langle R_{3}\right\rangle^{s t}\right] e^{-\Gamma_{p o p} t}+\left\langle R_{3}\right\rangle^{s t} .
\end{gathered}
$$

Here we have introduced the decay rates for the atomic populations and the atomic coherences, respectively [18]:

$$
\begin{gathered}
\Gamma_{p o p}=A_{-}+A_{+}, \\
\Gamma_{c o h}=\frac{4 A_{0}+A_{-}+A_{+}}{2}, \\
\left\langle R_{3}\right\rangle^{s t}=\frac{A_{-}-A_{+}}{A_{-}+A_{+}},
\end{gathered}
$$

and used the shorthand notations $R_{21}=R_{+}$and $R_{12}=R_{-}$.

As shown in Ref. [9], when the electromagnetic density of states varies abruptly with the frequency, the population rate of the dressed excited state $|\widetilde{2}\rangle$ may become smaller than its depopulation rate. In this case, there are values of the Rabi frequency for which the dressed state $|\widetilde{2}\rangle$ is less populated in the steady-state regime. In turn, this determines an accumulation of atomic population on the bare excited state $|2\rangle$, and the atomic system becomes inverted. This occurs when

$$
A_{+}>A_{-} \quad \Rightarrow \gamma_{+} c^{4}>\gamma_{-} s^{4} .
$$

The atomic switching threshold is obtained from the equation $A_{+}=A_{-}$. The resulting threshold Rabi frequency can be expressed as 


$$
\frac{\epsilon_{t h r}}{\left|\Delta_{A L}\right|}=\frac{\sqrt[4]{\gamma_{+} \gamma_{-}}}{\sqrt{\gamma_{+}}-\sqrt{\gamma_{-}}}
$$

Remarkably, the threshold Rabi frequency does not depend on the decay rate $\gamma_{0}$, nor on the dephasing rate $\gamma_{p}$.

In this Markov approximation [9], the magnitude of inversion is smaller than in the non-Markovian treatment [8], and the threshold laser intensity required is greater. Also, the switching threshold is not sharply defined. On the other hand, sharpness and magnitude of atomic switching can be greatly enhanced [7,9], if the single atom is replaced by a collection of $(N \gg 1)$ identical atoms, all confined to a cubic wavelength.

\section{FLUORESCENCE SPECTRUM NEAR A PHOTONIC BAND EDGE}

\section{A. The scattered radiation spectrum}

In this section, we evaluate the fluorescence spectrum of the atomic system. The steady-state spectrum radiated by the driven atomic system is defined as

$$
S(\omega)=\underset{t \rightarrow \infty}{q \lim \operatorname{Re}}\left[\int_{0}^{\infty} e^{-i\left(\omega-\omega_{L}\right) \tau}\left\langle\mathcal{E}^{-}(t+\tau) \mathcal{E}^{+}(\tau) d \tau\right\rangle\right],
$$

where

$$
\begin{aligned}
\mathcal{E}^{+}(t)= & i \sum_{\mathbf{k}, \lambda} \sqrt{\frac{\hbar \omega_{\mathbf{k}}}{2 e_{0} \mathcal{V}}} \mathbf{e}_{\mathbf{k}, \lambda} a_{\mathbf{k}, \lambda}(t) \\
= & \frac{i}{2 \epsilon_{0} \mathcal{V}} e^{-i \omega_{L} t} \sum_{\mathbf{k}, \lambda} \omega_{\mathbf{k}} \mathbf{e}_{\mathbf{k}, \lambda}\left(\mathbf{e}_{\mathbf{k}, \lambda} \cdot \mathbf{d}_{21}\right) \\
& \times \int_{0}^{t} d t^{\prime} \sigma_{12}\left(t^{\prime}\right) e^{\left(\omega_{\mathbf{k}}-\omega_{L}\right)\left(t-t^{\prime}\right)}
\end{aligned}
$$

$\mathcal{E}^{ \pm}(t)\left(\mathcal{E}^{-}(t)=\left[\mathcal{E}^{-}(t)\right]^{\dagger}\right)$ are the positive and negative frequency component operators of the scattered electric field [20], and $q$ is a constant containing geometric and atomic factors [21]. In Eq. (3.2) $a_{\mathbf{k}, \lambda}\left(a_{\mathbf{k}, \lambda}^{\dagger}\right)$ is the annihilation (creation) operator of a reservoir mode with wave vector $\mathbf{k}$ and polarization $\lambda$, $\mathbf{e}_{\mathbf{k}, \lambda}$ is the polarization of this mode, $\omega_{\mathbf{k}}$ corresponds to an arbitrary dispersion relation, and $\mathbf{d}_{21}$ is the dipole moment of the atomic transition. In the ordinary vacuum case $[\omega(\mathbf{k})=c k]$, the positive and negative frequency components of the scattered electric field become proportional to the atomic dipole operators, $\sigma_{ \pm}(t)$. However, in the case of a photonic crystal, the atomic dipole moment has components which encounter different photonic density of state, and, implicitly, have different radiative rates [20] $\left(\gamma_{0}, \gamma_{-}, \gamma_{+}\right)$. In particular,

$$
\sigma_{-} \equiv \sigma_{12}(t)=c s R_{3} e^{-i \omega_{L} t}-s^{2} R_{12} e^{-i\left(\omega_{L}-2 \Omega\right) t}+c^{2} R_{21} e^{-i\left(\omega_{L}+2 \Omega\right) t} .
$$

As a result, the operator of the scattered electric field has the form

$$
\begin{gathered}
\mathcal{E}^{+}(t)=A\left[\sqrt{\frac{\gamma_{0}}{2}} c s R_{3}(t)+\sqrt{\frac{\gamma_{-}}{2}} c^{2} e^{-2 i \Omega t} R_{12}(t)\right. \\
\left.-\sqrt{\frac{\gamma_{+}}{2}} s^{2} e^{2 i \Omega t} R_{21}(t)\right] \\
\mathcal{E}^{-}(t)=\left[\mathcal{E}^{-}(t)\right]^{\dagger}
\end{gathered}
$$

where $A$ is a frequency-independent constant.

In the presence of realistic nonradiative dephasing, the off-diagonal steady-state elements of the density operator vanish. The scattered radiation spectrum may then be separated into elastic and inelastic components

$$
S(\omega)=S_{e l}(\omega)+S_{i n}(\omega)
$$

where

$$
S_{e l}(\omega)=q \gamma_{0} c^{2} s^{2} \operatorname{Re}\left[\int_{0}^{\infty} d \tau e^{-i\left(\omega-\omega_{L}\right) \tau}\left\langle R_{3}(\tau)\right\rangle_{s}^{2}\right],
$$

and

$$
S_{\text {in }}(\omega)=S_{0}(\omega)+S_{-}(\omega)+S_{+}(\omega) \text {, }
$$

with

$$
\begin{gathered}
S_{0}(\omega)=q \gamma_{0} c^{2} s^{2} \operatorname{Re}\left[\int_{0}^{\infty} d \tau e^{-i\left(\omega-\omega_{L}\right) \tau}\left\langle R_{3}(\tau)\left(R_{3}-\left\langle R_{3}\right\rangle_{s}\right)\right\rangle_{s}\right], \\
S_{-}(\omega)=q \gamma_{-} s^{4} \operatorname{Re}\left[\int_{0}^{\infty} d \tau e^{-i\left(\omega-\omega_{L}+2 \Omega\right) \tau}\left\langle R_{12}(\tau) R_{21}\right\rangle_{s}\right], \\
S_{+}(\omega)=q \gamma_{+} c^{4} \operatorname{Re}\left[\int_{0}^{\infty} d \tau e^{-i\left(\omega-\omega_{L}-2 \Omega\right) \tau}\left\langle R_{21}(\tau) R_{12}\right\rangle_{s}\right] .
\end{gathered}
$$

Here the index $s$ indicates averages over the steady-state distribution, and the atomic operators without argument refer to the $\tau=0$ values.

We assume that the photonic DOS surrounding the dressed state frequencies $\omega_{L}-2 \Omega, \omega_{L}, \omega_{L}+2 \Omega$ is smoothly varying (Markov model). In this case, we utilize the quantum regression theorem [22] to evaluate the correlation functions $\left\langle O_{1}(t) O_{2}\right\rangle_{s}$, for $O_{1}$ and $O_{2}$ arbitrary atomic operators. The quantum regression theorem facilitates the evaluation of twotime averages by using equations that have the same structure as the ones obeyed by one-time operator averages. The two-operator products appearing in the spectrum [Eqs. (3.8)] are then given by [compare with Eq. (2.15a)]

$$
\begin{gathered}
\left\langle R_{ \pm}(\tau) R_{\mp}\right\rangle_{s}=\left\langle R_{ \pm} R_{\mp}\right\rangle_{s} e^{-\Gamma_{c o h} \tau}, \\
\left\langle R_{3}(\tau)\left(R_{3}-\left\langle R_{3}\right\rangle_{s}\right)\right\rangle_{s}=\left\langle R_{3}\left(R_{3}-\left\langle R_{3}\right\rangle_{s}\right)\right\rangle_{s} e^{-\Gamma_{p o p} \tau},
\end{gathered}
$$

with $\Gamma_{\text {pop,coh }}$ given by Eq. (2.16a). Performing the time integral in Eq. (3.8) yields 


$$
\begin{gathered}
S_{e l}(\omega)=\frac{q \pi}{2} \gamma_{0} c^{2} s^{2}\left[1-4 \pi_{2} \pi_{1}\right] \delta\left(\omega-\omega_{L}\right), \\
S_{0}(\omega)=2 q \pi \gamma_{0} c^{2} s^{2} \pi_{2} \pi_{1} \frac{\Gamma_{p o p}}{\Gamma_{p o p}^{2}+\left[\omega-\omega_{L}\right]^{2}}, \\
S_{-}(\omega)=\frac{q \pi}{2} \gamma_{-} s^{4} \pi_{1} \frac{\Gamma_{c o h}}{\Gamma_{c o h}^{2}+\left[\omega-\left(\omega_{L}-2 \Omega\right)\right]^{2}}, \\
S_{+}(\omega)=\frac{q \pi}{2} \gamma_{+} c^{4} \pi_{2} \frac{\Gamma_{c o h}}{\Gamma_{c o h}^{2}+\left[\omega-\left(\omega_{L}+2 \Omega\right)\right]^{2}},
\end{gathered}
$$

where we introduced the shorthand notations $\pi_{2}=\left\langle R_{21} R_{12}\right\rangle_{s}$ $=\left\langle R_{22}\right\rangle_{s}$ and $\pi_{1}=\left\langle R_{12} R_{21}\right\rangle_{s}=\left\langle R_{11}\right\rangle_{s}$. The inelastic scattered radiation exhibits a Mollow spectrum, with three bands, centered at the frequencies $\omega_{L}, \omega_{L}-2 \Omega$, and $\omega_{L}+2 \Omega$. The distinction from ordinary vacuum is found in the details of the peak heights and widths.

In the sharply colored vacuum of a photonic crystal, the spectral characteristics of the scattered radiation change considerably. While maintaining the three-peaked structure, the heights and the widths of the spectral components are changed from their free space values. In the absence of the phonon mediated dephasing, the symmetry of the Mollow spectrum is preserved, despite the asymmetry of the underlying density of states.

This spectral symmetry is consistent with the detailed balance condition (valid in the absence of the nonradiative dephasing mechanisms), which states that

$$
\pi_{1} \Gamma_{1 \rightarrow 2}=\pi_{2} \Gamma_{2 \rightarrow 1},
$$

where $\pi_{1}$ and $\pi_{2}$ denote the stationary populations of the dressed lower and upper states, respectively. $\Gamma_{1 \rightarrow 2}$ and $\Gamma_{2 \rightarrow 1}$ are the transition rates between dressed states $|\widetilde{1}\rangle \rightarrow|\widetilde{2}\rangle$ and $|\widetilde{2}\rangle \rightarrow|\widetilde{1}\rangle$, respectively. They are given by

$$
\begin{aligned}
& \Gamma_{1 \rightarrow 2}=\gamma_{-}\left|\left\langle\tilde{2}\left|\sigma_{+}\right| \tilde{1}\right\rangle\right|^{2}=\gamma_{-} s^{4}, \\
& \Gamma_{2 \rightarrow 1}=\gamma_{+}\left|\left\langle\tilde{1}\left|\sigma_{+}\right| \tilde{2}\right\rangle\right|^{2}=\gamma_{+} c^{4} .
\end{aligned}
$$

In the absence of dipolar dephasing $\left(\gamma_{p} / \gamma_{+}=0\right)$, the equilibrium dressed-state populations [Eqs. (2.16a)] are given by

$$
\begin{aligned}
& \pi_{2}=\frac{\gamma_{-} s^{4}}{\gamma_{-} s^{4}+\gamma_{+} c^{4}}, \\
& \pi_{1}=\frac{\gamma_{+} c^{4}}{\gamma_{-} s^{4}+\gamma_{+} c^{4}},
\end{aligned}
$$

and the detailed balance condition, Eq. (3.12), is verified. The weight of a sideband is given by the number of photons emitted per second in this line, which, in turn, is determined by the product between the population of the specific atomic state and the corresponding transition probability. The detailed balance condition [Eq. (3.12)] expresses the fact that the sidebands have the same spectral weight. The left sideband is determined by transitions originating from the dressed state $|\tilde{1}\rangle$, and has a spectral weight of $\pi_{1} \Gamma_{1 \rightarrow 2}$, whereas the right sideband appears as a result of transition from the dressed state $|\tilde{1}\rangle$, and has a spectral weight of $\pi_{2} \Gamma_{2 \rightarrow 1}$. It follows from Eq. (3.12) that in the absence of other (nonradiative) dephasing mechanisms, the spectrum of the scattered radiation is symmetrical regardless of the frequency dependence of the photonic reservoir density of states.

On the other hand, spectral asymmetry arises in the presence of nonradiative dephasing [18]. This breakdown of the detailed balance condition can be associated with the nonradiative redistribution of system energy among different states caused by the interaction with an additional external reservoir. In this case, the system (atom $\otimes$ driving field $\otimes$ photonic reservoir) becomes an open system. Dipolar dephasing can transfer the atom from a dressed state to another dressed state without the emission of a photon. This process results in a redistribution of the population among different dressed states, which, in turn, affects the relative magnitude of the spectral components of the emitted radiation. In the presence of dipolar dephasing, the weight of the left sideband is given by

$$
W_{\omega_{L}-2 \Omega}=\pi_{1} \Gamma_{1 \rightarrow 2}=\gamma_{-} s^{4} \frac{\gamma_{+} c^{4}+4 \gamma_{p} c^{2} s^{2}}{\gamma_{+} s^{4}+\gamma_{+} c^{4}+8 \gamma_{p} c^{2} s^{2}},
$$

whereas the weight of the right sideband is now given by

$$
W_{\omega_{L}+2 \Omega}=\pi_{2} \Gamma_{2 \rightarrow 1}=\gamma_{+} c^{4} \frac{\gamma_{-} s^{4}+4 \gamma_{p} c^{2} s^{2}}{\gamma_{+} s^{4}+\gamma_{+} c^{4}+8 \gamma_{p} c^{2} s^{2}} .
$$

Clearly, the relative weight of the sideband components can be controlled by variations in the driving field intensity. For example, the right sideband weight becomes larger than the weight of its left counterpart $\left(W_{\omega_{L}+2 \Omega}>W_{\omega_{L}-2 \Omega}\right)$ for Rabi frequencies for which $\gamma_{+} c^{4}>\gamma_{-} s^{4}$. This is precisely the inversion threshold condition investigated in Ref. [9]. For negative detunings of the atomic frequency $\left(\Delta_{A L}<0\right)$ and below the threshold, $\epsilon<\epsilon_{t h r}$ [see Eq. (2.18)], the weight of the left sideband is larger than its right counterpart (abovethreshold behavior is the mirror image of below-threshold behavior). This influence of the dipolar dephasing on the relative weight of the spectral components is presented in Figs. 5 and 6. We also note that for ordinary vacuum, for negative detunings, the weight of the left sideband is always larger than the weight of the right sideband (since in this case $\gamma_{+} c^{4}>\gamma_{-} s^{4}$, for any value of $\epsilon$ ).

We now discuss the specific influence of the photonic DOS discontinuity on the spectral characteristics of the radiation emitted by the atomic system. In Fig. 7, we plot the peak height of the central Mollow component as a function of the Rabi frequency $\epsilon /\left|\Delta_{A L}\right|$ and the jump in the photonic DOS $\gamma_{-} / \gamma_{+}$, for negative detunings $\Delta_{A L}$, and no dipolar dephasing in the system. We obtain that, instead of the monotonic saturation of the spectral intensity with the ap- 


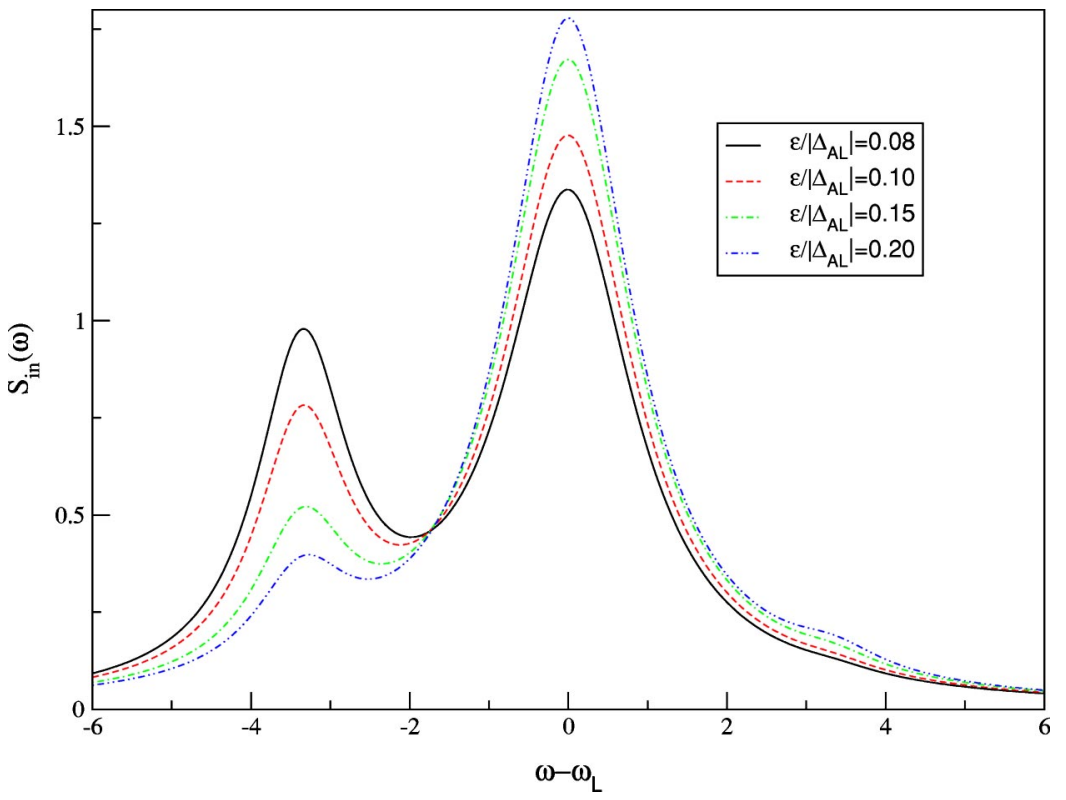

FIG. 5. Scattered radiation spectrum as a function of the detuning frequency, $\Delta=\omega-\omega_{L}$ (in units of $\gamma_{+}$), for different values of the Rabi frequency in the presence of dipolar dephasing. All Rabi frequencies used here are below the threshold value, which, for the specific choice of the parameters used in this figure, is $\varepsilon_{t h r} / \Delta_{A L}=0.35$. The atomic system is negatively detuned from the laser field frequency, $\left|\Delta_{A L}\right| / \gamma_{+}=3$, and $\gamma_{-} / \gamma_{+}$ $=0.01$. The dipolar dephasing rate is $\gamma_{p} / \gamma_{+}=0.1$. plied laser field obtained in the ordinary vacuum case, the switching effect in a photonic crystal is accompanied by a resonance like behavior in the central Mollow line (more pronounced for small ratios $\left.\gamma_{-} / \gamma_{+}\right)$. Also, while the central component is enhanced in the immediate vicinity of the switching threshold, the sidebands are strongly suppressed. This characteristic feature of the switching threshold appears also in an exact multiphoton treatment of resonance fluorescence in colored vacuum $[17,19]$. The dependence of the widths of the spectral components, $\Gamma_{\text {pop,coh }}$, on the decay constant ratio $\gamma_{-} / \gamma_{+}$can also be inferred from Eq. (2.16a). All of the spectral components exhibit a slight narrowing for large jumps in the photonic density of states. Finally, we remark that the nonradiative dephasing has a deleterious influence on the Mollow spectrum, by strongly reducing the heights and increasing the widths of the individual components.

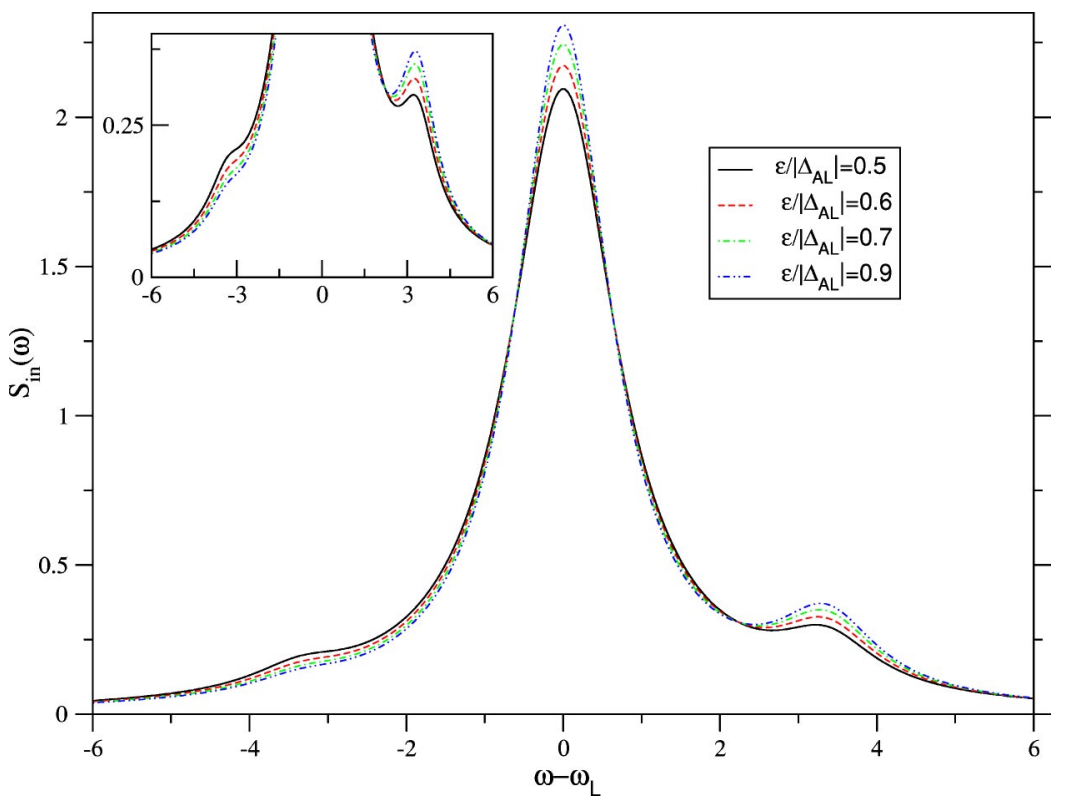

\section{B. Photon antibunching}

The measurements of photon correlation functions have become a major tool in quantum optics and the spectroscopy of single-quantum systems [23]. The second-order correlation function of the light scattered from the resonant atom gives the probability of detecting two photons with a temporal separation of $\tau$ [16], and is defined by

$$
G^{(2)}(\tau) \propto\left\langle\mathcal{E}^{(-)}(0) \mathcal{E}^{(-)}(\tau) \mathcal{E}^{(+)}(\tau) \mathcal{E}^{(+)}(0)\right\rangle_{s}
$$

The normalized version of this correlation function is the degree of second-order coherence of the scattered field,

$$
g^{(2)}(\tau)=\frac{G^{(2)}(\tau)}{\lim _{\tau \rightarrow \infty} G^{(2)}(\tau)}
$$

FIG. 6. Scattered radiation spectrum in the presence of dipolar dephasing, as a function of the detuning frequency, $\Delta=\omega-\omega_{L}$ (in units of $\left.\gamma_{+}\right)$, for different values of the Rabi frequency. All Rabi frequencies used here are above the threshold value, which, for the specific choice of the parameters used in this figure, is $\epsilon_{t h r} / \Delta_{A L}$ $=0.35$. The atomic system is negatively detuned from the laser field frequency $\left|\Delta_{A L}\right| / \gamma_{+}=3$, and $\gamma_{-} / \gamma_{+}=0.01$. The dipolar dephasing rate is $\gamma_{p} / \gamma_{+}=0.1$. The inset shows an expanded (on the $y$ axis) view of the sideband peak region. 


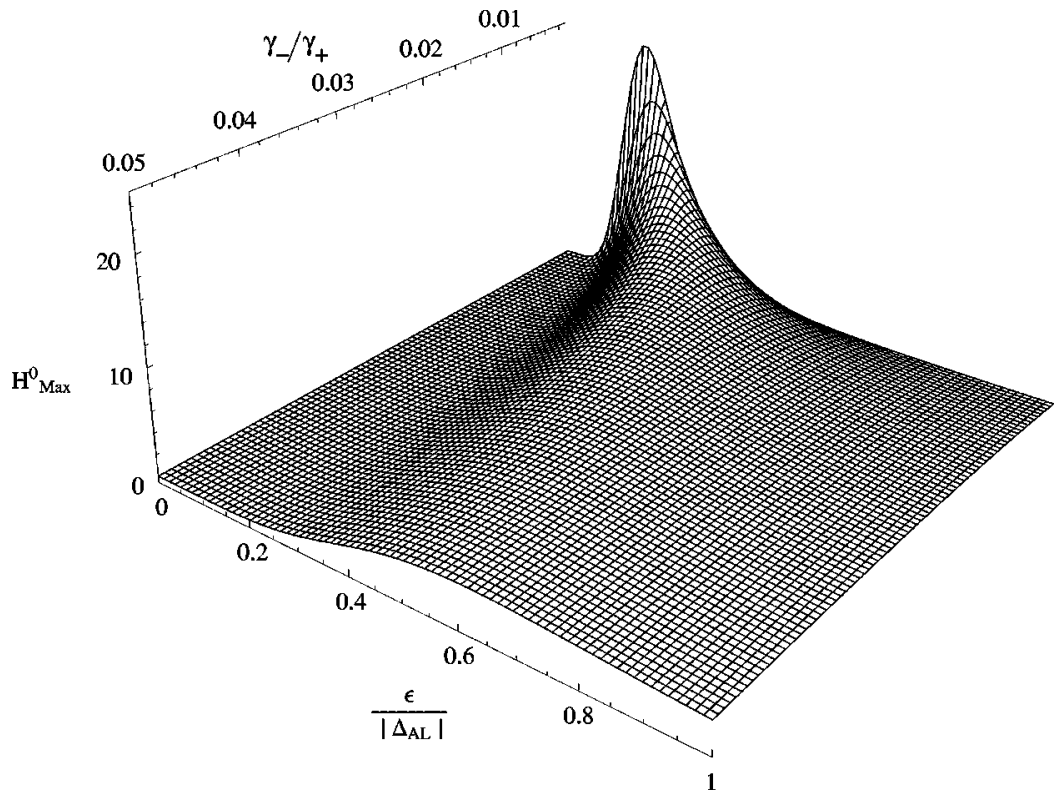

FIG. 7. The peak height $H_{\max }^{0}$ of the central component of the scattered spectrum (in arbitrary units) as a function of the Rabi frequency $\epsilon /\left|\Delta_{A L}\right|$ for $\left|\Delta_{A L}\right| / \gamma_{+}=3, \operatorname{sgn}\left(\Delta_{A L}\right)=-1$, and the magnitude of the jump in the photonic DOS, $\gamma_{-} / \gamma_{+}$, in the absence of the dipolar dephasing, $\gamma_{p} / \gamma_{+}=0$. The atomic resonant frequency is detuned negatively from the laser field frequency $\Delta_{A L}=\omega_{A}$ $-\omega_{L}<0$.

In this section, we evaluate the second-order correlation function of the light scattered from the resonant atom coupled to the radiation reservoir of a photonic crystal. In principle, the evaluation of the degree of second-order coherence (in the Markovian formalism used in this paper) does not bring with it new theoretical difficulties nor does it require new approximations: it follows a similar path to the evaluation of the spectral properties of the scattered light presented in the preceding section. Since the off-diagonal elements of the density matrix vanish in the stationary limit, four operator products in Eq. (3.17) can be simplified using the quantum regression theorem. We obtain after a tedious but straightforward calculation (see the Appendix for details)

$$
\begin{aligned}
g^{(2)}(\tau)= & 1-2 \gamma_{0} e^{-\Gamma_{c o h} \tau} \cos (2 \Omega \tau) \\
& \times \frac{\sqrt{\gamma_{-}} \sqrt{\gamma_{+}} c^{2} s^{2}+\gamma_{+} c^{4} \pi_{2}+\gamma_{-} s^{4} \pi_{1}}{\left(\gamma_{0}^{2}+\gamma_{-} \gamma_{+}\right) c^{2} s^{2}+2 \gamma_{0}\left(\gamma_{+} c^{4} \pi_{2}+\gamma_{-} s^{4} \pi_{1}\right)} .
\end{aligned}
$$

In ordinary vacuum case $\left(\gamma_{0}=\gamma_{+}=\gamma_{-}\right)$and in the absence of dipolar dephasing, $\gamma_{p h}=0$, this reduces to

$$
g^{(2)}(\tau)=1-e^{-\Gamma_{c o h} \tau} \cos (2 \Omega \tau),
$$

where now $\Gamma_{c o h}=\gamma / s\left(1+2 s^{2} c^{2}\right)$. In the on-resonance case $\left(\Delta_{A L}=\omega_{L}-\omega_{A}=0\right) s^{2}=c^{2}=1 / 2$, and we obtain the usual strong-field limit of the second-order correlation function

$$
g^{(2)}(\tau)=1-e^{-3 / 4 \gamma \tau} \cos (2 \varepsilon \tau) .
$$

In Fig. 8, we plot $g^{(2)}(\tau)$ for more general Rabi frequency, $\epsilon /\left|\Delta_{A L}\right|$, in the ordinary vacuum case, and in the absence of dipolar dephasing. The second-order correlation function exhibits the well-known antibunching character, $\lim _{\tau \rightarrow 0} g^{(2)}(\tau)$ $=0$ [16], which shows that the probability to simultaneously detect two photons (time separation $\tau=0$ ) vanishes and the photons tend to scatter individually. As shown in Fig. 9, for small temporal separations, the probability to simultaneously detect two photons may increase slightly in a photonic crys- tal. Also, the second-order coherence function shows important features, such as a larger amplitude of oscillations and a slower rate of decay of the oscillatory behavior. In Fig. 10, we show that the probability to simultaneously detect two photons, $g^{(2)}(0)$, increases with the density of states jump $\gamma_{+} / \gamma_{-}$. While for ratios $\gamma_{-} / \gamma_{+}$close to unity, the strong $\left[g^{(0)}(0)=0\right]$ antibunching effect characterizing the ordinary vacuum case is regained, for larger jumps in the photonic DOS, the scattered photons exhibit a vanishingly small antibunching character. In the limit of a full band gap in the photonic DOS, $\gamma_{-} / \gamma_{+}=0$, the second-order coherence function reaches unity, i.e., the atom becomes a source of fully coherent radiation (in this regime [20], the atom scatters photons only elastically, similar to a perfect classical scatterer). A physical interpretation of these effects is provided below. In the presence of dipolar dephasing (Fig. 11), while the second-order correlation function maintains similar features, important details emerge, such as a nontrivial dependence on the applied field Rabi frequency.

The dressed-state diagram together with the spectral properties of the scattered radiation from the dressed system (analyzed in Sec. III) provide a simple explanation of the photon antibunching effects described above. In the ordinary vacuum case, the correlations between the emitted photons (which give rise to the antibunching effect) have two equivalent interpretations. In the bare picture, after the atom has spontaneously emitted a photon, it takes a certain amount of time to be reexcited (by the absorption of a laser field photon). Thus two spontaneous emission events are separated by a finite time interval and the probability to detect simultaneously two photons is zero. In the dressed-state picture, the correlations among emitted photons are apparent as well.

The bare (dressed) picture diagram is presented on the left (right) panel of Fig. 12. We denote the state in which the atom is in its $i$ th state $(i=1,2)$ and there are $n$ photons in the background by the ket vector $|i, n\rangle$. The splitting between the states $|1, n+1\rangle$ and $|2, n\rangle$ is given by the detuning between the atomic and laser frequency, $\Delta_{A L}=\omega_{A}-\omega_{L}$. The states 


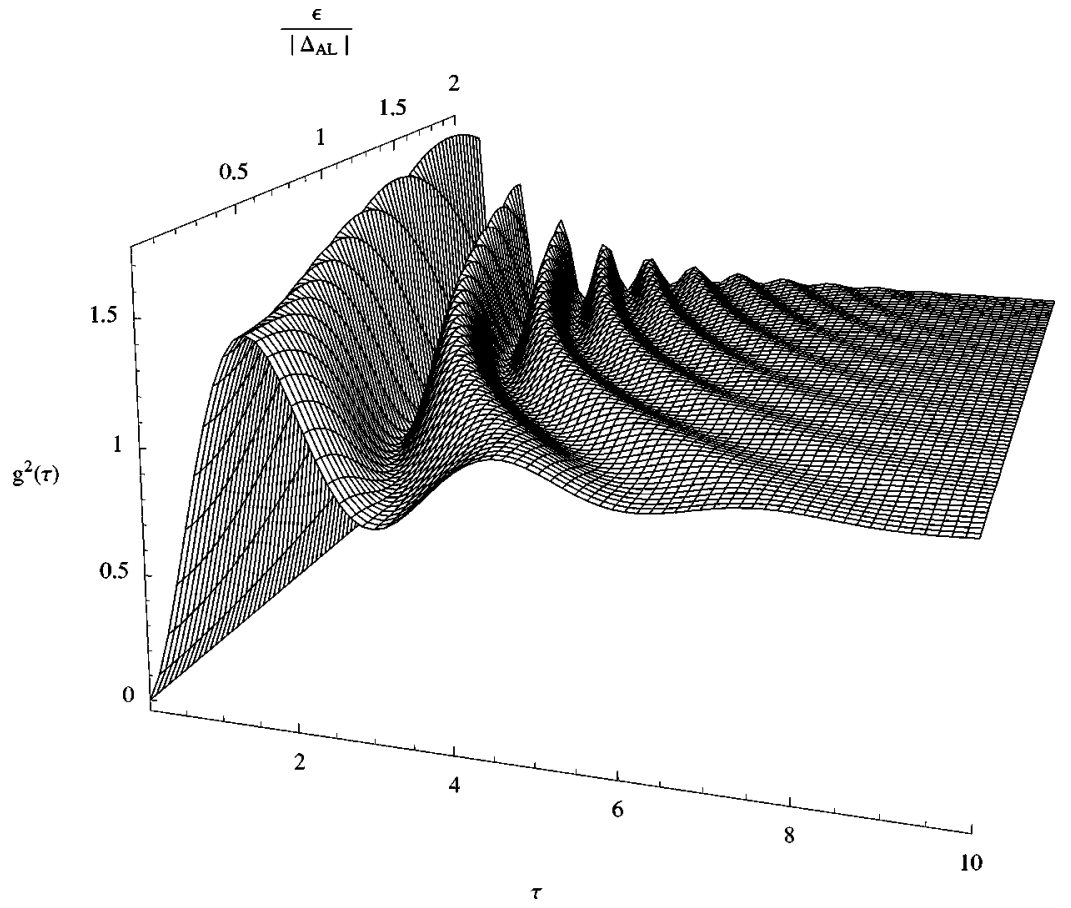

FIG. 8. Second-order correlation function $g^{(2)}(\tau)$ as a function of the scaled time $\tau=\gamma t$ and the Rabi laser field frequency, $\epsilon /\left|\Delta_{A L}\right|$, for ordinary vacuum case $\gamma_{-} / \gamma_{+}=\gamma_{0}$, and in the absence of the dipolar dephasing, $\gamma_{p} / \gamma_{+}=0$. The atomic resonant frequency is detuned negatively from the laser field frequency, $\Delta_{A L}=\omega_{A}-\omega_{L}<0$.

$|1, n+1\rangle$ and $|2, n\rangle$ are coupled by the laser field-atom interaction Hamiltonian $H_{A L}$, and we associate the interaction matrix element with the Rabi frequency $\epsilon:\left\langle 2, n\left|H_{A L}\right| 1, n+1\right\rangle$ $\approx \hbar \varepsilon$. Provided that $\Delta_{A L}$ and $\varepsilon$ are small compared to $\omega_{L}$, we can neglect all the couplings between different atom $\otimes$ laser manifolds, characterized by different number of photons in the laser field, $n$ (see left panel of Fig. 12).

If the "atom" is initially in the state $|\tilde{2}, n\rangle$ on the left panel of Fig. 12, then, it can only decay by the emission of a "high" photon (at the frequency $\omega_{L}+2 \Omega$ ) or a "medium" photon (at the frequency $\omega_{L}$ ). These processes place the atom in either state $|\tilde{1}, n-1\rangle$ or state $|\tilde{2}, n-1\rangle$. Assume now the atom is in the state $|\tilde{1}, n-1\rangle$. Then it can decay by either emitting a "low" photon (at the frequency $\omega_{L}-2 \Omega$ ) or a "medium" photon. In the first case the atom will decay into the state $|\tilde{2}, n-2\rangle$, whereas in the second case will decay in state $|\tilde{1}, n-2\rangle$. This is the dressed picture of the so called "radiative cascade." Clearly, between the emissions of two high (low) photons, there must be an emission of a low (high) photon. These correlations between high and low photons have been investigated theoretically [18] and have been observed experimentally [25]. In this picture, the only uncorrelated photons are the medium photons, which contribute to the central (both elastic and inelastic) components of the

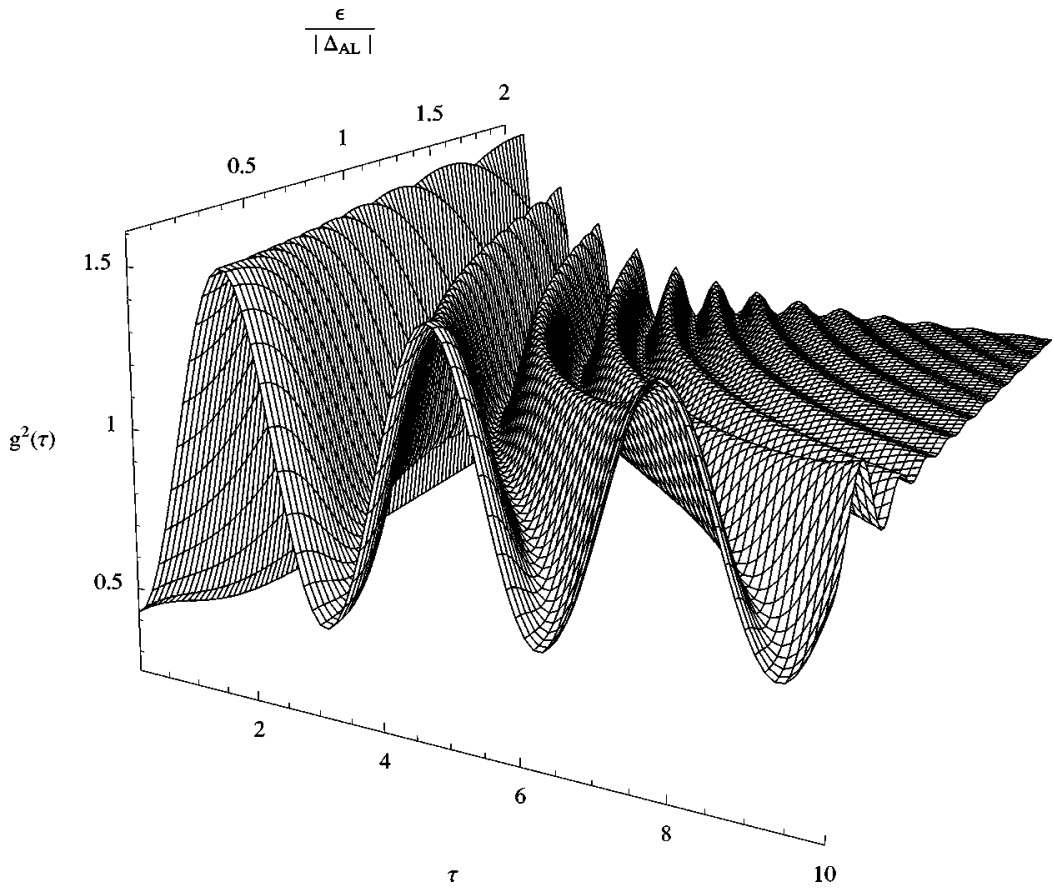

FIG. 9. Second-order correlation function $g^{(2)}(\tau)$ as the function of the scaled time $\tau=\gamma_{+} t$ and the Rabi laser field frequency, $\epsilon /\left|\Delta_{A L}\right|$, for a photonic crystal characterized by $\gamma_{-} / \gamma_{+}=0.001$, $\gamma_{0} / \gamma_{+}=1$, and in the absence of the dipolar dephasing, $\gamma_{p} / \gamma_{+}=0$. The atomic resonant frequency is detuned negatively from the laser field frequency, $\Delta_{A L}=\omega_{A}-\omega_{L}<0$. 


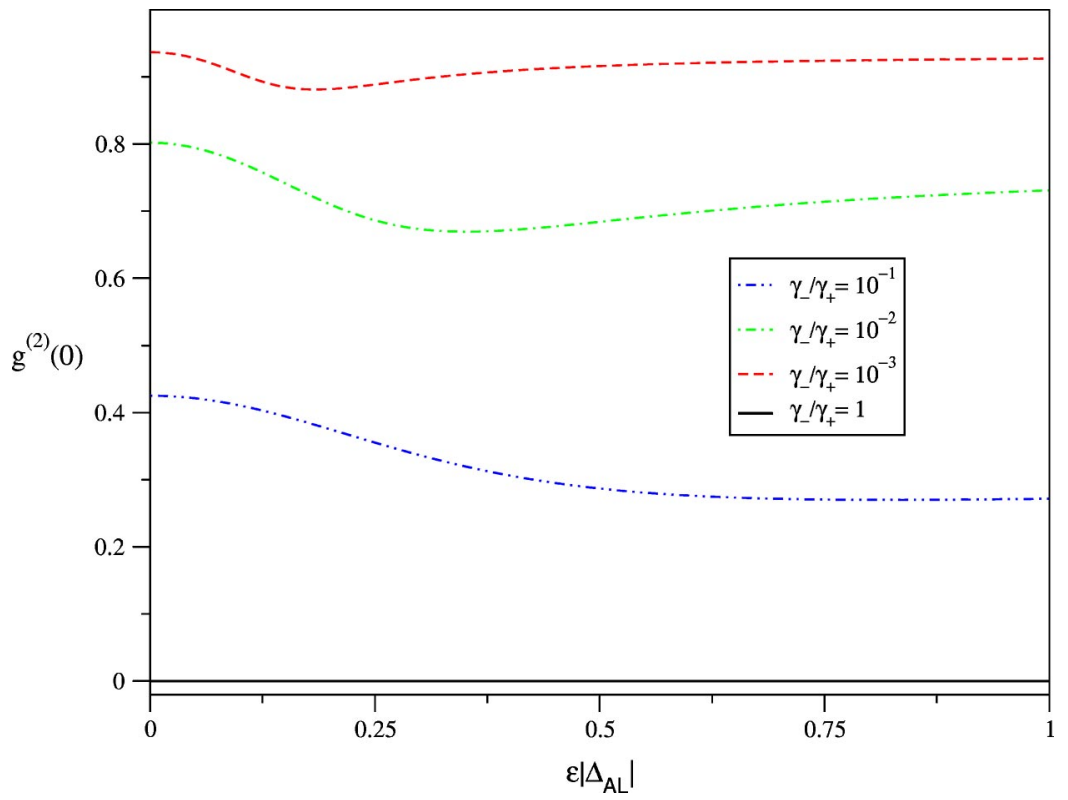

FIG. 10. Second-order correlation function $g^{(2)}(0)$ as a function of the Rabi laser field frequency, $\epsilon /\left|\Delta_{A L}\right|$, in the absence of the dipolar dephasing, $\gamma_{p} / \gamma_{+}=0$, for different magnitudes of the jump in the photonic DOS. The atomic resonant frequency is detuned negatively from the laser field frequency, $\Delta_{A L}=\omega_{A}-\omega_{L}<0$.

Mollow spectrum. In the presence of the photonic crystal, we show that the decay rates involving low and high photons suffer an important reduction. Therefore, the emission will consist primarily of medium photons among which there are no correlations (in the dressed emission cascade, there are no restrictions about the subsequent emission of medium photons). In the case of a full photonic band gap $\left(\gamma_{-}=0\right)$, the only possible emission process is that of medium photons (the probability of high and low photon emission vanishes if $\left.\gamma_{-}=0\right)$. Therefore, in this case, there are no correlations between the emitted photons [20] and $g^{(2)}(0)=1$.

\section{Collective atomic effects and ultrafast atomic switching}

We consider a collection of $N$ identical two-level atomic systems with a transition frequency near a discontinuous change in the electromagnetic DOS coherently driven by an external laser field. We assume the atoms are (i) confined to a volume smaller than the cube of the radiation wavelength or (ii) placed in a lattice of nearly equivalent positions in a photonic crystal. Consequently, the atoms experience the same field amplitude (Dicke model). We also assume that the atom-laser field coupling is strong compared to any direct dipole-dipole interaction between atoms.

In Ref. [7] it was shown that, for large jumps in the photonic DOS, the atomic ensemble exhibits collective switching and atomic inversion without fluctuations. The width of the switching region is of order $O(1 / N)$. This implies that for a large number of atoms, the switching effect is extremely sudden (i.e., the required modulation in the intensity of the external driving field is very small).

The spectral properties of the scattered radiation from the atomic ensemble can be obtained analogously to that of the single atom. In the Heisenberg equation of motion approach,

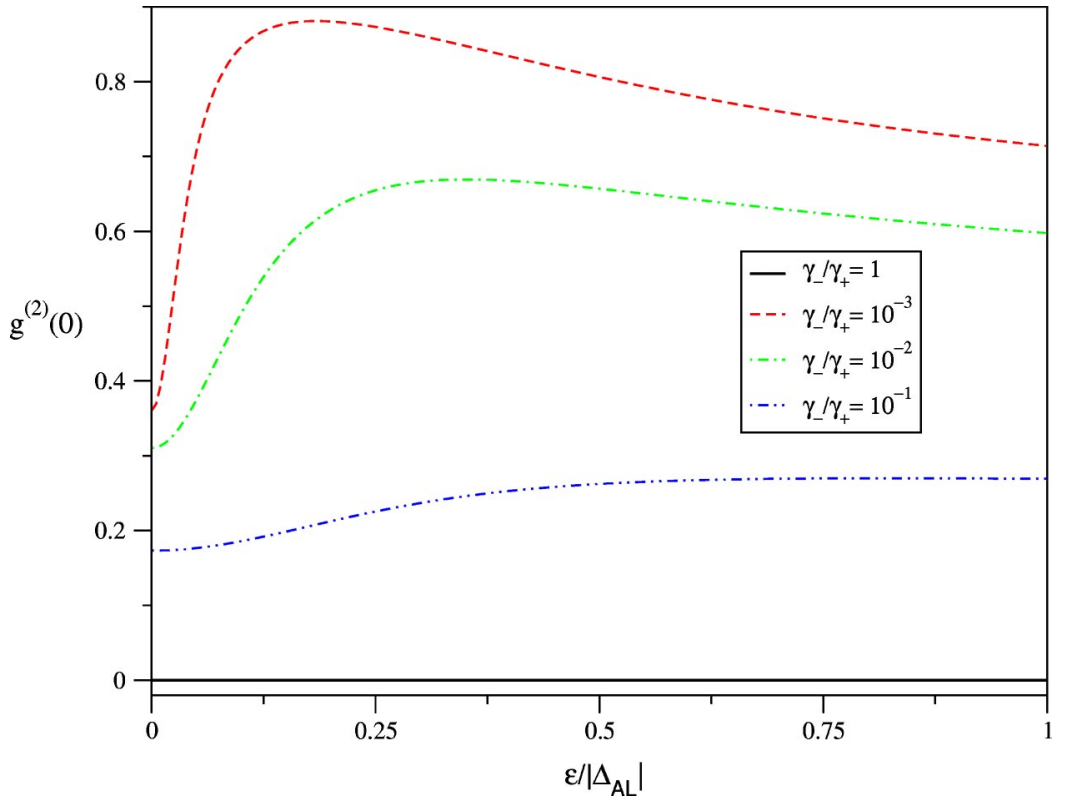

FIG. 11. Second-order correlation function $g^{(2)}(0)$ as a function of the Rabi laser field frequency, $\epsilon /\left|\Delta_{A L}\right|$, in the presence of the dipolar dephasing, $\gamma_{p} / \gamma_{+}=0.2$, for different magnitudes of the jump in the photonic DOS. The atomic resonant frequency is detuned negatively from the laser field frequency, $\Delta_{A L}=\omega_{A}-\omega_{L}<0$. 


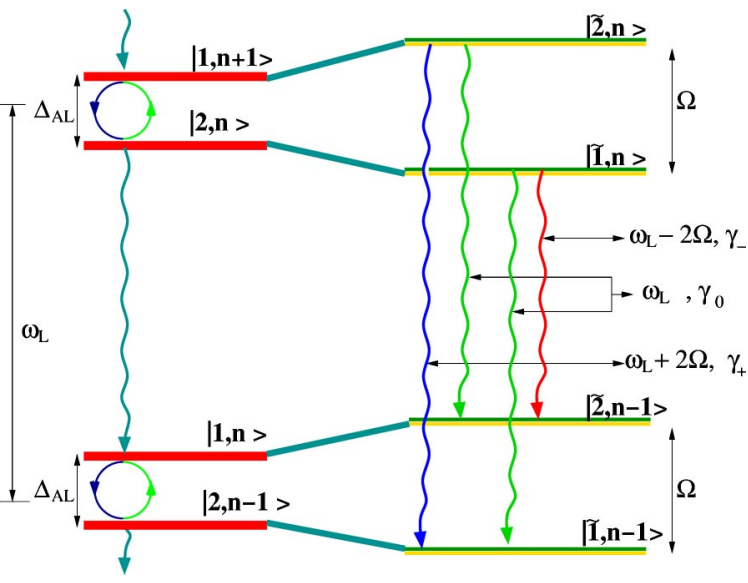

FIG. 12. Bare atom and dressed atom states diagram.

the time rate of change of any product of $k$ collective atomic operators involves products of $k+1$ atomic operators. This leads to a hierarchy of equations that contain an everincreasing number of atomic operators. In order to solve this system of equations for the operator expectation values, one can either assume a relatively small number of atoms in the ensemble and deal with it numerically, or introduce a suitable factorization scheme (mean-field approximation) for the product of atomic operators [24]. Both approaches have their own merits and drawbacks. The numerical approach can be used to investigate the whole parameter space of the problem, but is limited to a relative small number of atoms. In the approach based on decorrelation schemes, the number of atoms can be arbitrary large, but the range of parameters is limited to the domain of validity of the mean-field approximation. In this section, we use a relatively simple decorrelation scheme, suggested by Compagno and Persico [24]. In a natural generalization of the single-atom resonance fluorescence problem, the reduced master equation that governs the evolution of the atomic collection is given by

$$
\frac{d \rho(t)}{d t}=\left.\frac{d \rho(t)}{d t}\right|_{\text {sec }}+\left.\frac{d \rho(t)}{d t}\right|_{ \pm 2 \Omega}+\left.\frac{d \rho(t)}{d t}\right|_{ \pm 4 \Omega},
$$

with

$$
\begin{aligned}
2\left[\frac{\partial \rho}{\partial t}\right]_{s e c}= & A_{0}\left[R_{3} \rho R_{3}-R_{3}^{2} \rho\right]+A_{-}\left[R_{21} \rho R_{12}-R_{12} R_{21} \rho\right] \\
& +A_{+}\left[R_{12} \rho R_{21}-R_{21} R_{12} \rho\right]+\text { H.c. }, \quad(3.22 \mathrm{~b}) \\
2\left[\frac{\partial \rho}{\partial t}\right]_{ \pm 2 \Omega}= & c s e^{-2 i \Omega t t}\left\{s^{2} \gamma_{0}\left[R_{12} \rho(t)-R_{3} \rho(t) R_{12}\right]\right. \\
& +c^{2} \gamma_{0}\left[R_{12} \rho(t) R_{3}+\rho(t) R_{12}\right]-c^{2} \gamma_{+}\left[R_{3} \rho(t) R_{12}\right. \\
& \left.\left.-\rho(t) R_{12}\right]+s^{2} \gamma_{-}\left[R_{12} \rho(t)-R_{12} \rho(t) R_{3}\right]\right\}+ \text { H.c. }
\end{aligned}
$$

$$
2\left[\frac{\partial \rho}{\partial t}\right]_{ \pm 4 \Omega}=c^{2} s^{2} e^{4 i \Omega t}\left(\gamma_{-}+\gamma_{+}\right) R_{12} \rho(t) R_{12}+\text { H.c. }
$$

The master equation (3.22) yields the following equations of motion for the scaled atomic operators $r_{i j} \equiv R_{i j} / N(i, j$ $=1,2)$, and $r_{3} \equiv R_{3} / N$,

$$
\begin{aligned}
2\left\langle\dot{r}_{21}(t)\right\rangle \equiv & 2 \operatorname{Tr}\left[\frac{\partial \rho(t)}{\partial t} r_{21}\right]=-\frac{4 A_{0}+A_{+}+A_{-}}{4}\left\langle r_{21}(t)\right\rangle \\
& +N \frac{A_{+}-A_{-}}{2}\left\langle\left\{r_{3}(t), r_{21}(t)\right\}\right\rangle \\
2\left\langle\dot{r}_{3}(t)\right\rangle \equiv & 2 \operatorname{Tr}\left[\frac{\partial \rho(t)}{\partial t} r_{3}\right]=\frac{A_{+}+A_{-}}{2}\left\langle r_{3}(t)\right\rangle+N\left(A_{+}-A_{-}\right) \\
& \times\left\langle\left\{r_{21}(t), r_{12}(t)\right\}\right\rangle=\frac{A_{+}+A_{-}}{2}\left\langle r_{3}(t)\right\rangle+N\left(A_{+}-A_{-}\right) \\
& \times\left[\left\langle r_{3}(t)^{2}\right\rangle-N(N+2)\right],
\end{aligned}
$$

where $\{A, B\}$ denotes the anticommutator of the operators $A$ and $B$. In the single-atom case $(N=1),\left\{r_{3}(t), r_{21}(t)\right\}$ $=0,\left\{r_{21}(t), r_{12}(t)\right\}=1$, etc., but for $N>1$, the hierarchy of equations needs to be closed by a factorization scheme.

The steady-state behavior of the collective atomic variables was analyzed in Refs. [7,9]. As in the single-atom case, for large deviations of the photonic mode density between the components of the scattered radiation spectrum (Mollow spectrum), the inversion in the atomic population occurs at low threshold. Unlike the single-atom case, for a large number of atoms in the cubic wavelength (or otherwise identically placed atoms), the collective switching from the ground state and the excited state becomes very sharp even within the Markov approximation. In particular, $N$-atom collective switching occurs over a very narrow range (of order $1 / N$ ) of intensities of the external laser field. Under certain conditions, the excited atoms exhibit strong sub-Poissonian statistics and the switching speed exhibits a collective enhancement proportional to $N$. The average number of atoms in the excited dressed state is given by [7]

$$
\langle n\rangle=\frac{(N+1) \xi^{N+1}}{\xi^{N+1}-1}-\frac{\xi}{\xi-1},
$$

where $\xi \equiv A_{-} / A_{+}$.

We start by analyzing the dynamics of the atomic operators in the immediate vicinity of the threshold intensity. For $\epsilon=\epsilon_{t h r}$, we have $A_{+}=A_{-}$, and the equations of motion become

$$
\begin{gathered}
2\left\langle\dot{r}_{21}(t)\right\rangle=-\frac{4 A_{0}+A_{+}+A_{-}}{4}\left\langle r_{21}(t)\right\rangle, \\
2\left\langle\dot{r}_{3}(t)\right\rangle=\frac{A_{+}+A_{-}}{2}\left\langle r_{3}(t)\right\rangle .
\end{gathered}
$$

The equations of motion for the dressed operators (3.25) are essentially linear and decoupled from each other. They admit a simple solution 


$$
\begin{aligned}
\left\langle r_{21}(t)\right\rangle & =\left\langle r_{21}(0)\right\rangle e^{-\Gamma_{c o h} t}, \\
\left\langle r_{3}(t)\right\rangle & =\left\langle r_{3}(0)\right\rangle e^{-\Gamma_{p o p} t},
\end{aligned}
$$

where $\Gamma_{\text {coh,pop }}$ are the single-atom widths of the spectral components calculated in Sec. III [see Eq. (2.16a)]. In this case, the dressed-atom collective variables are damped to zero on time scales corresponding to the single-atom spontaneous decay constants. The response time scale of the collective atomic system (given by the inverse of the decay rates $\left.\Gamma_{\text {coh,pop }}\right)$ is the same as in the single-atom case. For atomic transitions in the optical regime, this is of the order of nanoseconds. Using the quantum regression theorem and the procedure developed in Sec. III (single-atom case), Eqs. (3.25) yield the scattered spectrum from $N$-atoms:

$$
S^{(N)}(\omega) \approx N^{2} S(\omega)
$$

where $S(\omega)$ is the single-atom spectrum calculated in Sec. III. The $N^{2}$ factor arises from the $1 / N$ scaling of the collective atomic operators. At the threshold intensity, the collective scattered spectrum consists of the three usual Mollow lines with widths $\Gamma_{c o h}^{(N)}=\Gamma_{c o h}$ (sideband components) and $\Gamma_{\text {pop }}^{(N)}=\Gamma_{\text {pop }}$ (central component). We conclude that in the threshold regime the shape of the spectrum remains unchanged from the single-atom case. However, the scattered intensity scales as $N^{2}$.

We now investigate the scattered spectrum outside the threshold regime. In this case, $A_{+} \neq A_{-}$and the solution of Eqs. (3.23a) will strongly depend on the number of atoms. In order to proceed, we use a relatively simple decoupling scheme [24], which corresponds to the approximation

$$
\left\langle\left\{r_{3}, r_{i j}\right\}\right\rangle=2\left\langle r_{3}\right\rangle_{s}\left\langle r_{i j}\right\rangle,
$$

where the \langle\rangle$_{s}$ denotes steady-state expectation values, and $i, j=1,2$. For simplicity, we concentrate on the sideband spectra. The equation of motion for $r_{21}$ is now given by

$$
2\left\langle\dot{r}_{21}(t)\right\rangle=-\left[\frac{4 A_{0}+A_{+}+A_{-}}{4}-N \frac{A_{+}+A_{-}}{2}\left\langle r_{3}\right\rangle_{s}\right]\left\langle r_{21}(t)\right\rangle .
$$

Here, we use the steady-state solution for the collective atomic inversion operator evaluated in the preceding section, $\left\langle r_{3}\right\rangle_{s}=2\langle n\rangle / N-1$.

The width of the sideband components of the scattered spectrum is then given by

$$
\Gamma_{c o h}^{(N)}=\Gamma_{c o h}+N\left(A_{+}-A_{-}\right)\left\langle r_{3}\right\rangle_{s} \approx N\left(A_{+}-A_{-}\right)\left\langle r_{3}\right\rangle_{s} .
$$

In contrast to the threshold regime, the sidebands are now considerably broadened, with widths proportional to the number of atoms. The sideband contributions to the scattered spectrum are given by

$$
S_{-}^{(N)}(\omega) \propto\left\langle R_{12} R_{21}\right\rangle_{s} \gamma_{-} s^{4} \frac{\Gamma_{c o h}^{(N)}}{\left(\Gamma_{c o h}^{(N)}\right)^{2}+\left[\omega-\left(\omega_{L}-2 \Omega\right)\right]^{2}},
$$

$$
S_{+}^{(N)}(\omega) \propto\left\langle R_{21} R_{12}\right\rangle_{s} \gamma_{+} c^{4} \frac{\Gamma_{c o h}^{(N)}}{\left(\Gamma_{c o h}^{(N)}\right)^{2}+\left[\omega-\left(\omega_{L}+2 \Omega\right)\right]^{2}} .
$$

Using the steady-state solution of the master equation (3.22), we obtain

$$
\begin{gathered}
\left\langle R_{12} R_{21}\right\rangle_{s}=(N-\langle n\rangle)(\langle n\rangle+1), \\
\left\langle R_{21} R_{12}\right\rangle_{s}=\langle n\rangle(N-\langle n\rangle+1),
\end{gathered}
$$

where the atomic population inversion $n$ is given by Eq. (3.24). The collective response time of the active medium to a secondary pump pulse is inversely proportional to the number of atoms. This suggests that, for appropriate concentrations of active atoms, the atomic switching could take place on sub-picosecond time scales, rather than the nanosecond time scale of a single atom.

\section{ABSORPTION AND GAIN SPECTRUM OF A WEAK PROBE BEAM}

\section{A. Single-atom optical susceptibility}

In this section, we consider that, in addition to the strong driving laser field, the atomic system is probed by a second laser field. The probe field is assumed to be weak so as not to disturb the dressed state of the atomic system. The linear susceptibility is given in terms of a field correlation function evaluated in the absence of the probe field [18,26,27]:

$$
\chi(\omega)=i A \int_{0}^{\infty}\left\langle\left[\mathcal{E}^{+}(t), \mathcal{E}^{-}\right]\right\rangle_{s} e^{i \omega t} d t
$$

where $A$ is a normalization constant, the index $s$ indicates that the average is evaluated in the steady-state limit, and the operators $\mathcal{E}^{ \pm}$[given by Eq. (3.4)] correspond to the positive and negative frequency components of the scattered electric field (similar to the scattered spectrum calculations presented in Sec. III A and Ref. [20], we define the linear susceptibility in terms of the field correlation function). In the strong-field limit, it can be shown [using Eq. (4.1) and the fact that the off-diagonal elements of the density matrix vanish in the stationary limit] that the central component of $\chi(\omega)$ at the frequency $\omega_{L}$ disappears from the absorption spectrum. The probe field "probes" the dressed transitions (presented on the right panel of Fig. 12), and the contribution of a given transition, say between the states $i$ and $j$, is proportional to $\left(\pi_{j}\right.$ $\left.-\pi_{i}\right) \Gamma_{j i}$. Here, $\pi_{i, j}$ denote the populations on the $i, j$ states and $\Gamma_{j i}$ is the probability of the transition $i \rightarrow j$. In general the absorption spectrum would (similar to the scattered spectrum) consist of three components at the Mollow components, $\omega_{L}, \omega_{L} \pm 2 \Omega$. The central line contains contributions from the transitions $|\tilde{1}, n\rangle \rightarrow|\tilde{1}, n-1\rangle,|\tilde{2}, n\rangle \rightarrow|\tilde{2}, n-1\rangle$, and so forth. However, in the case of large number of photons (large $\epsilon$ ), the dressed diagram becomes periodic, i.e., the states $|\tilde{i}, n\rangle$ and $|\tilde{i}, n-1\rangle$ have roughly the same population. As a result, the central component of the absorption signal (proportional to the population difference between these states) vanishes in this limit. The linear susceptibility has 


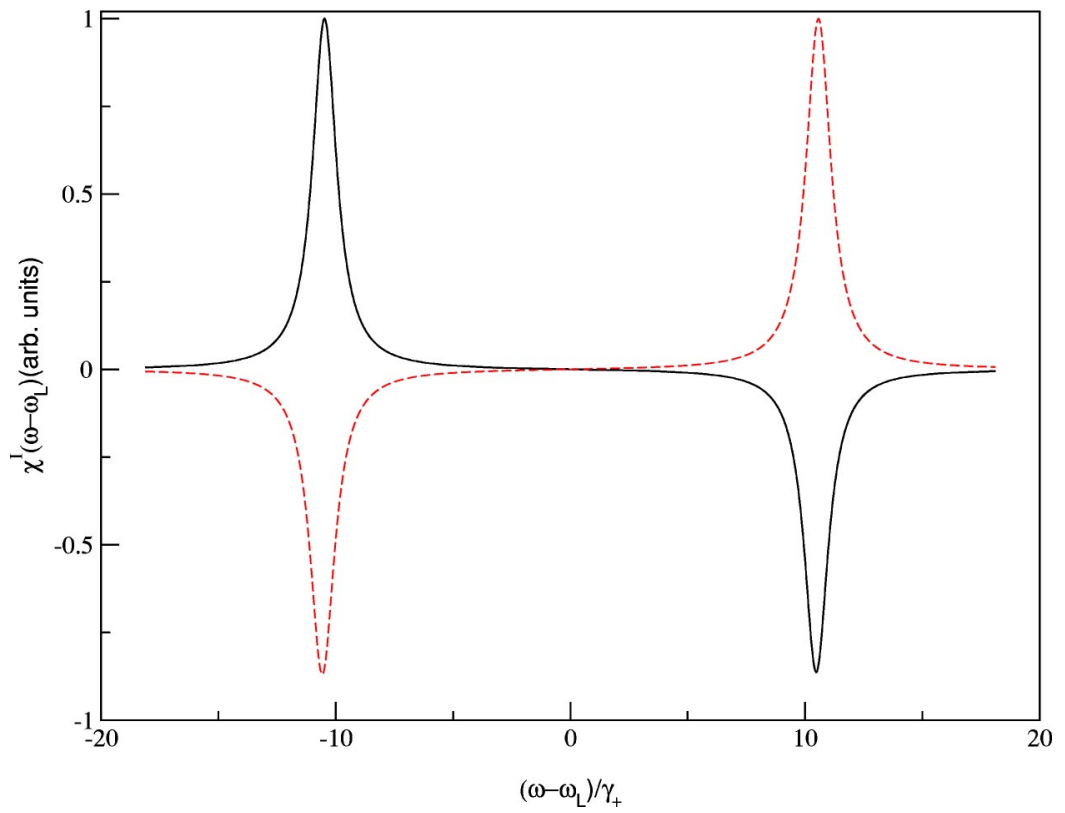

FIG. 13. The imaginary part of the linear susceptibility of the probe beam (absorption spectrum) as a function of the detuning of the probe beam frequency from the driving laser field frequency $\omega-\omega_{L}$, for $\gamma_{-} / \gamma_{+}=0.001, \quad \gamma_{0} / \gamma_{+}=1$, $\gamma_{p} / \gamma_{+}=0.2,\left|\Delta_{A L}\right| / \gamma_{+}=10$. The two curves correspond to different Rabi frequencies, $\epsilon /\left|\Delta_{A L}\right|$, one of them (continuous curve) below the inversion threshold $\left(\epsilon /\left|\Delta_{A L}\right|=0.176\right)$, and the other one (dashed curve) above the threshold intensity $\left(\epsilon /\left|\Delta_{A L}\right|=0.190\right)$. The atomic resonant frequency is detuned negatively from the laser field frequency, $\operatorname{sgn}\left(\Delta_{A L}\right)=-1$, and, for this choice of parameters, the inversion threshold is $\epsilon_{t h r} /\left|\Delta_{A L}\right|$ $=0.183$.

two well separated components, at the frequencies $\omega_{L} \pm 2 \Omega$ [21],

$$
\chi(\omega)=\chi_{(+)}(\omega)+\chi_{(-)}(\omega) .
$$

Using Eq. (3.4), these components are given by

$$
\begin{aligned}
& \chi_{(+)}(\omega)=i A \gamma_{+} c^{4} \int_{0}^{\infty}\left\langle\left[R_{12}(t), R_{21}\right]_{s}\right\rangle e^{i\left[\omega-\left(\omega_{L}+2 \Omega\right)\right] t} d t, \\
& \chi_{(-)}(\omega)=i A \gamma_{-} s^{4} \int_{0}^{\infty}\left\langle\left[R_{21}(t), R_{12}\right]_{s}\right\rangle e^{i\left[\omega-\left(\omega_{L}-2 \Omega\right)\right] t} d t .
\end{aligned}
$$

The correlation function present in Eq. (4.3) is then evaluated using the quantum regression theorem in a manner analogous to that used to derive Eq. (3.6). We obtain

$$
\begin{aligned}
& {\left[R_{21}(t), R_{12}\right]=\left[R_{21}, R_{12}\right] e^{-\Gamma_{c o h} t},} \\
& {\left[R_{12}(t), R_{21}\right]=\left[R_{12}, R_{21}\right] e^{-\Gamma_{c o h} t} .}
\end{aligned}
$$

Here, for simplicity we have omitted the zero-time argument of the atomic operators $R_{k} \equiv R_{k}(0)$. Finally, the real and imaginary parts of components of the linear susceptibility $\chi(\omega)$ are cast in the form

$$
\begin{aligned}
& \chi_{(+)}^{R}(\omega)=A \quad \gamma_{+} c^{4}\left(\pi_{2}-\pi_{1}\right) \frac{\omega-\left(\omega_{L}+2 \Omega\right)}{\left[\omega-\left(\omega_{L}+2 \Omega\right)\right]^{2}+\Gamma_{c o h}^{2}}, \\
& \chi_{(-)}^{R}(\omega)=A \quad \gamma_{-} s^{4}\left(\pi_{1}-\pi_{2}\right) \frac{\omega-\left(\omega_{L}-2 \Omega\right)}{\left[\omega-\left(\omega_{L}-2 \Omega\right)\right]^{2}+\Gamma_{c o h}^{2}},
\end{aligned}
$$

$$
\chi_{(+)}^{I}(\omega)=-A \gamma_{+} c^{4}\left(\pi_{2}-\pi_{1}\right) \frac{\Gamma_{c o h}}{\left[\omega-\left(\omega_{L}+2 \Omega\right)\right]^{2}+\Gamma_{c o h}^{2}},
$$

$$
\chi_{(-)}^{I}(\omega)=-A \gamma_{-} s^{4}\left(\pi_{1}-\pi_{2}\right) \frac{\Gamma_{c o h}}{\left[\omega-\left(\omega_{L}-2 \Omega\right)\right]^{2}+\Gamma_{c o h}^{2}} .
$$

The spectrum experienced by the probe beam (given by the imaginary part of the linear susceptibility) consists of one absorptive sideband and one amplifying sideband [note the sign difference between the components in Eqs. (4.5)]. The discontinuous photonic DOS of the photonic crystal opens a possibility of controlling the absorptive or amplifying character of the components through small variations in the intensity of the driving laser field. The main difference between the fluorescence spectrum and the absorption spectrum [as seen from Eqs. (3.11) and (4.5)] is that the absorption signal is proportional to the difference of the population between the dressed levels involved in the transition, whereas the fluorescent signal depends only on the population of the initial state of the transition (see also Refs. [18,28]). Clearly, the character of the absorption signal components is determined by the sign of the dressed atomic inversion, which, in turn, is determined by the intensity of the driving field.

In both free space and the photonic crystal, one sideband is absorptive and the other sideband is amplifying (negative absorption). However, in the photonic crystal (as opposed to ordinary vacuum), the magnitude of the amplifying component is comparable to that of the absorbing component and is readily detectable in a pump-probe spectroscopy experiment. The spectral separation of the two components is proportional to the intensity of the driving field and their relative magnitude can be optimized by adjusting the driving field intensity. In particular, as shown in Fig. 13, the absorptive or amplifying character of the sidebands becomes extremely 


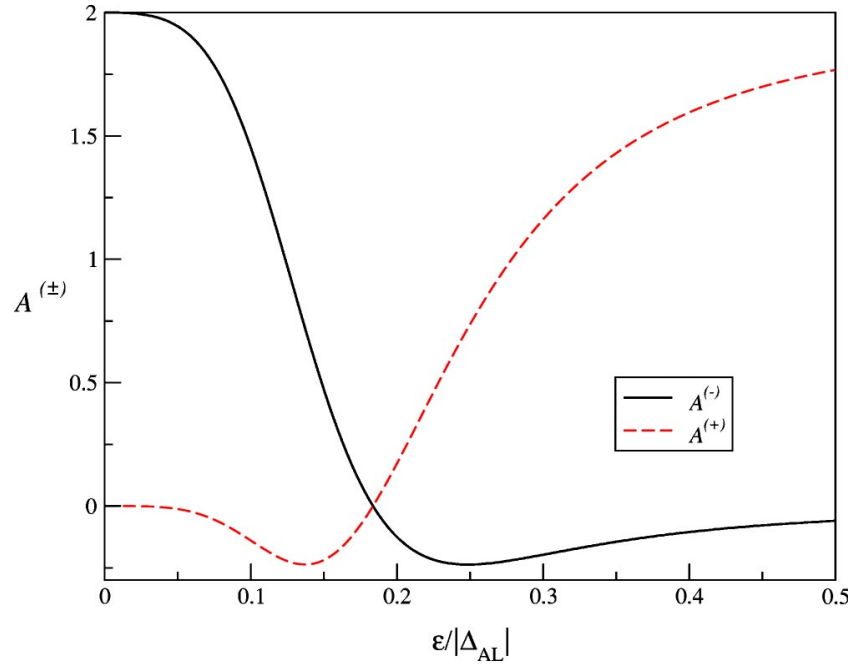

FIG. 14. The peak height to width ratio [Eq. (4.6)] of the components of the absorption spectrum of the probe field as a function of the Rabi frequency of the driving field $\epsilon /\left|\Delta_{A L}\right|$, for $\gamma_{-} / \gamma_{+}$ $=0.001, \gamma_{0} / \gamma_{+}=0.001$, and in the absence of the dipolar dephasing, $\gamma_{p} / \gamma_{+}=0$. The atomic resonant frequency is detuned negatively from the laser field frequency, $\Delta_{A L}=\omega_{A}-\omega_{L}<0$.

sensitive to variations in the driving laser field near the switching threshold intensity $\epsilon_{t h r}$.

The frequency-dependent character of the photonic reservoir facilitates the inversion of the atomic population (in the bare picture) and the switching of the linear response (of the dressed system) to a weak probe laser field.

The predicted gain spectrum can be optimized by appropriate choices of the control parameters. To facilitate this optimization process, we introduce the peak height to width ratio value as a measure of the efficiency of the amplification process

$$
A^{ \pm} \equiv \frac{W_{\omega_{L} \pm 2 \Omega}^{a b s}}{\Gamma_{c o h}}
$$

where $W_{\omega_{L} \pm 2 \Omega}^{a b s}$ are the weights of the sideband spectral components $\left[W_{\omega_{L}+2 \Omega}^{a b s}=\left(\pi_{1}-\pi_{2}\right) \Gamma_{1 \rightarrow 2} \quad\right.$ and $\quad W_{\omega_{L}-2 \Omega}^{a b s}=\left(\pi_{2}\right.$ $\left.\left.-\pi_{1}\right) \Gamma_{2 \rightarrow 1}\right]$ and $\Gamma^{( \pm)}=\Gamma_{c o h}$ is their width. As we show in Fig. 14, the peak height to width ratio of the components of the probe beam absorption spectrum (a measure of the efficiency of the amplification) reaches a maximum of $24 \%$ (the negative minima in Fig. 14), about four times larger than the ordinary vacuum value [28]. In Fig. 15, we compare the widths of the absorption spectrum sidebands as a function of the Rabi driving field laser frequency for the photonic crystal and ordinary vacuum. Clearly, the sideband components of the spectrum are appreciably narrowed in the presence of a strong jump in the DOS of the photonic crystal.

\section{B. Collective atomic effects and gain broadening}

The evaluation of the absorption spectrum of a probe beam for a collection of $N$ atoms follows closely the derivation of the fluorescence spectrum presented in Sec. III C. We obtain for the sideband components

$$
\begin{aligned}
\chi_{(-)}^{(N, R)}(\omega) \propto & \left\langle\left[R_{12} R_{21}, R_{21} R_{12}\right]\right\rangle_{s} \gamma_{-} s^{4} \\
& \times \frac{\Gamma_{c o h}^{(N)}}{\left(\Gamma_{c o h}^{(N)}\right)^{2}+\left[\omega-\left(\omega_{L}-2 \Omega\right)\right]^{2}}, \\
\chi_{(+)}^{(N, R)}(\omega) \propto & \left\langle\left[R_{21} R_{12}, R_{12} R_{21}\right]\right\rangle_{s} \gamma_{+} c^{4} \\
& \times \frac{\Gamma_{c o h}^{(N)}}{\left(\Gamma_{c o h}^{(N)}\right)^{2}+\left[\omega-\left(\omega_{L}+2 \Omega\right)\right]^{2}},
\end{aligned}
$$

with

$$
\left\langle\left[R_{12} R_{21}, R_{21} R_{12}\right]\right\rangle_{s}=-(2\langle n\rangle-N)=-\left\langle R_{3}\right\rangle,
$$

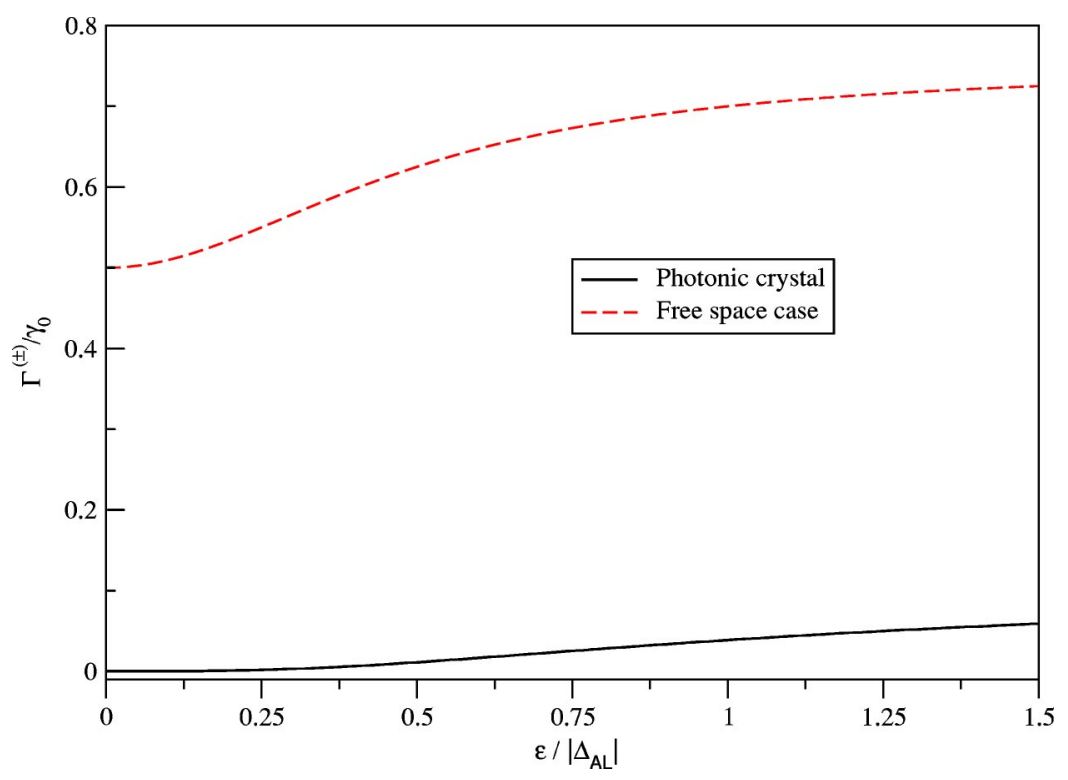

FIG. 15. The widths of the components of the absorption spectrum of the probe field in the presence of a photonic crystal, $\Gamma_{c o h}^{P C}$, and in free space $\Gamma_{c o h}^{F S}$, as a function of the Rabi frequency of the driving laser field $\epsilon /\left|\Delta_{A L}\right|$, for $\gamma_{-} / \gamma_{+}=0.001$, $\gamma_{0} / \gamma_{+}=0.001$, and in the absence of the dipolar dephasing, $\gamma_{p} / \gamma_{+}=0$. The atomic resonant frequency is detuned negatively from the laser field frequency, $\Delta_{A L}=\omega_{A}-\omega_{L}<0$. 


$$
\left\langle\left[R_{21} R_{12}, R_{12} R_{21}\right]\right\rangle_{s}=2\langle n\rangle-N=\left\langle R_{3}\right\rangle .
$$

Clearly, the switching and amplification of the probe field exhibit collective enhancement. The gain spectrum is broadened by a factor of $N$ relative to the single-atom spectral width, and the driving field modulation required to switch the system from the absorbing to the amplifying regime is reduced by a factor of $N$ [the width of the transition region is of order $\mathcal{O}(1 / N)]$.

\section{SWITCHING IN AN INHOMOGENEOUSLY BROADENED ATOMIC SYSTEM}

The application of the population inversion effect to an all-optical switch or transistor device will invariably require an ensemble of atoms (quantum dots) interacting resonantly with coherent laser fields [9]. Such an ensemble will involve spatial distribution (and size distribution of quantum dots) of resonators which experience different local environments or which have different inherent resonance frequencies. This will lead to an inhomogeneous broadening of the spectrum that may be very useful if the optical device is intended to have a gain spectrum that covers a telecommunication band of $60 \mathrm{~nm}$. On the other hand, random detunings of the atomic resonance from the photonic band edge (discontinuity in the density of states) may wash out the overall switching effect, since individual atomic switching is very sensitive to the detuning. In this paper, we discuss in detail the effects of inhomogeneous line broadening on the probe beam spectrum and switching effect. We describe how further electromagnetic density of states engineering within a PBG (combined with photobleaching techniques to remove those absorbers that degrade the switching effect) can be effectively utilized to both offset the deleterious effects of inhomogeneous broadening and provide an enlarged all-optical switching bandwidth. We explicitly show that, under certain conditions, the amplification and the switching of a probe beam can be fully recovered even in the presence of a broad distribution of atomic resonance frequencies (Fig. 16).

\section{A. Weak inhomogeneous broadening}

Consider an active medium consisting of a gas of trapped atoms in the void regions of a PBG crystal. Such an ensemble will exhibit a small amount of inhomogeneous broadening caused by the Doppler effect. For the sodium $3 S_{1 / 2}$ $\rightarrow 3 P_{3 / 2}$ transition, in free space, at room temperature, and for a density of $\mathcal{N}=6 \times 10^{14}$ atoms $/ \mathrm{cm}^{3}$, typical experimental quantities are [29] $\gamma=0.031 \mathrm{GHz}, \Delta_{A L}=1.5 \mathrm{GHz}$, and $\Omega$ $=2.6 \mathrm{GHz}$. The width of the Doppler broadened atomic line shape is $\Delta \omega_{I}=7.14 \mathrm{GHz}\left(\Delta \omega_{I} \approx 5 \Delta_{A L}\right)$. For the corresponding cooled atomic system, we use $\Delta \omega_{I} \approx 0.1-\left|\Delta_{A L}\right|$. In Fig. 17 , we plot the absorption spectrum as a function of the probe field frequency for inhomogeneous broadening in the range $\Delta \omega_{I} /\left|\Delta_{A L}\right|=0.1-0.9$. Clearly, not only does the amplifying (absorbing) component become wider, but it also develops an absorbing (amplifying) wing, whose relative weight increases with $\Delta \omega_{I}$. This wing can be easily traced to the threshold condition for the atomic inversion. In the presence of random detunings $\Delta_{A L}$, the effective threshold for

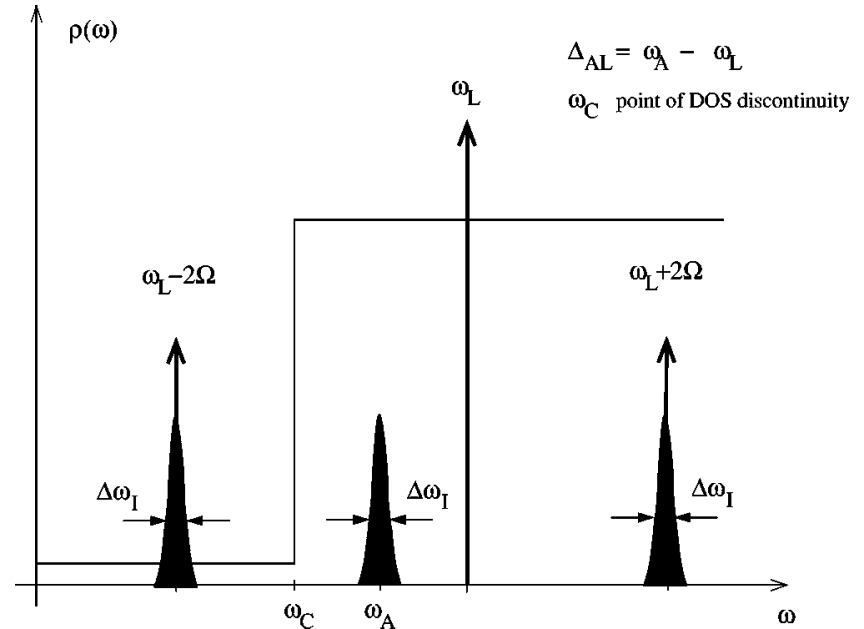

FIG. 16. Weakly inhomogeneously broadened Mollow spectrum near a steplike discontinuity in the electromagnetic density of states. We assume that the density of states is constant over spectral ranges larger than the inhomogeneous linewidth $\Delta \omega_{I}$, surrounding the Mollow triplet frequencies $\omega_{L}, \omega_{L} \pm 2 \Omega$.

inversion $\left(\epsilon_{t h r} /\left|\Delta_{A L}\right|\right)$ varies randomly from atom to atom. Therefore, instead of a sharp amplifying (absorbing) peak, we obtain a smeared feature. Moreover, some members of the ensemble may contribute oppositely from others, changing their character from amplifying (absorbing) to absorbing (amplifying). As clearly seen in Fig. 18, this mutual cancellation effect is less severe away from the switching threshold intensity of the driving field, and the absorption/gain features are simply broadened. However cold atom systems may be impractical for many important applications.

To conclude, small amounts of inhomogeneous broadening may improve the switching characteristics by enlarging the spectral range over which the switching occurs. Practical applications, however, may require systems that operate at or above room temperature. In general, this leads to stronger broadening as discussed below.

\section{B. Vacuum engineering in the case of strong inhomogeneous broadening}

If the active medium consists of quantum dot layers in a planar waveguide sandwiched inside a 3D PBG crystal, inhomogeneous broadening arising from the size distribution of dots may be considerably larger than for cold, trapped, atoms. In this case, a simple step discontinuity in the photon density of states is generally insufficient to realize an overall switching effect on the probe susceptibility. As a simple estimate, the inhomogeneous broadening present in ensemble of quantum dots may be no smaller than few percent of the central frequency, i.e., on the order of tens of $\mathrm{THz}$ (covering roughly $75 \mathrm{~nm}$ of bandwidth). In this case, additional engineering of the vacuum density of states is required to recapture a transistor function. We discuss below the required photonic crystal density of states architectures for this situation.

\section{Density of states engineering within the photonic band gap}

By suitable engineering of the photonic DOS, it is possible to quench those parts of the inhomogeneous broadened 


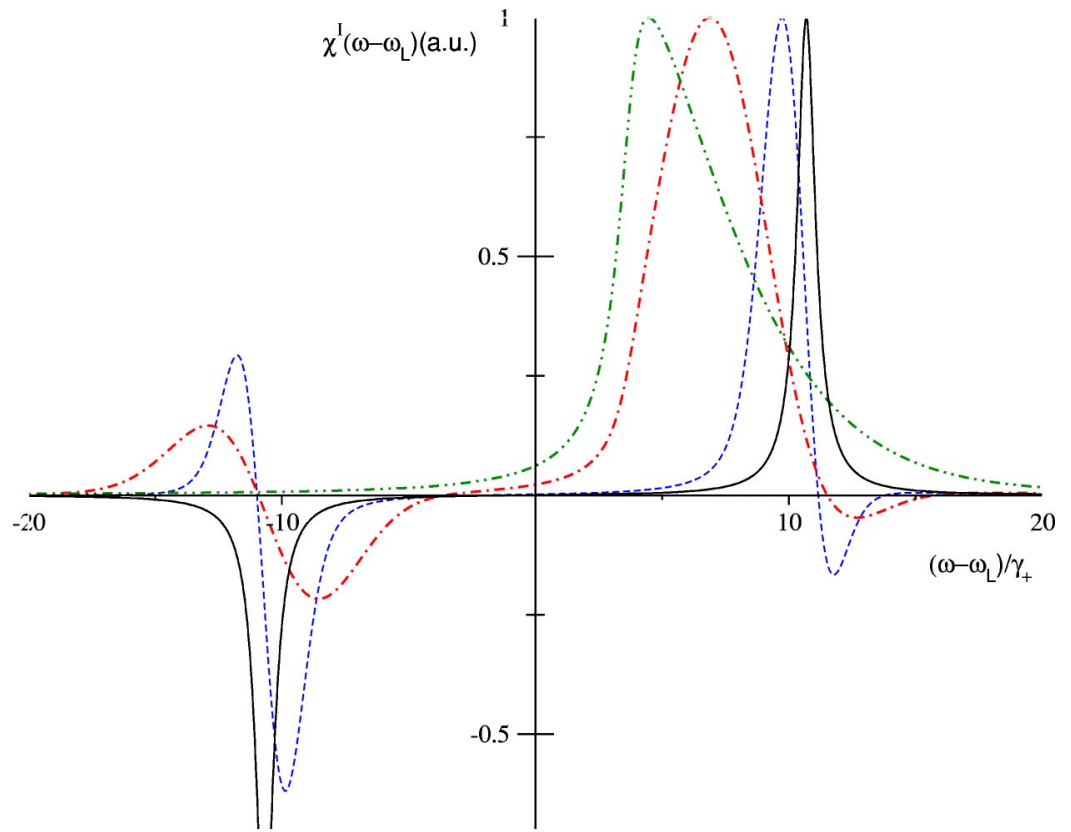

FIG. 17. The imaginary part of the linear susceptibility of the probe beam (absorption spectrum) as a function of the detuning of the probe beam frequency $\omega$ with respect to the driving laser field frequency $\omega_{L}$, for $\gamma_{-} / \gamma_{+}=0.001, \gamma_{0} / \gamma_{+}$ $=1, \epsilon /\left|\Delta_{A L}\right|=0.178$ and in the absence of the phonon mediated dephasing $\left(\gamma_{p} / \gamma_{+}=0\right)$. The width of the inhomogeneous line distribution varies from $\Delta \omega_{I} /\left|\Delta_{A L}\right|=0$ (no inhomogeneous broadening) for the solid curve to $\Delta \omega_{I} /\left|\Delta_{A L}\right|=0.9$ for the double-dot-dashed curve. The center of the atomic frequency distribution is detuned negatively from the laser field frequency, $\Delta_{A L}=\omega_{A}$ $-\omega_{L}<0$.

atomic distribution which hinder the overall switching effect. The ideal photonic DOS is presented in Fig. 19. The upper diagram depicts the simple steplike DOS (the staircase curve) used in the absence of the inhomogeneous broadening. The solid curves depict the evolution of the Mollow sideband peaks for a particular quantum dot, as its deviation $\Delta \omega_{A}$ from the center of the inhomogeneous distribution is increased. This evolution is simply given by:

$$
\omega_{L} \pm 2 \Omega=\omega_{L} \pm 2 \sqrt{\epsilon^{2}+\frac{\left(\Delta_{A L}+\Delta \omega_{A}\right)^{2}}{4}} .
$$

We note that when $\Delta_{A L}$ and $\Delta \omega_{A}$ have opposite signs, the trajectory of the sideband undergoes a reversal at $\omega_{L} \pm 2 \Omega_{M I N}$.
The engineered photonic DOS presented in the lower part of Fig. 19 provides a filter effect on the very broad inhomogeneous atomic distribution. It introduces a new frequency scale $\Omega_{M I N}$ that represents the effective width of the active ensemble that contributes to amplification. Atoms with resonant frequencies farther away from the center of the distribution than this given spectral distance cannot couple to either the driving field or the probe field. This scale is determined by the robustness of the switching process with respect to detuning $\Delta \omega_{A}$. As an example, for the parameters used in the preceding section, the switching effect survives for $\Delta \omega_{A}$ up to $20 \%$ of the threshold intensity value $\epsilon_{t h r}$. Accordingly, we choose $\Omega_{M I N}=0.2 \epsilon_{t h r}$. This atomic quenching effect relies crucially on the ability to microfabricate the ap-

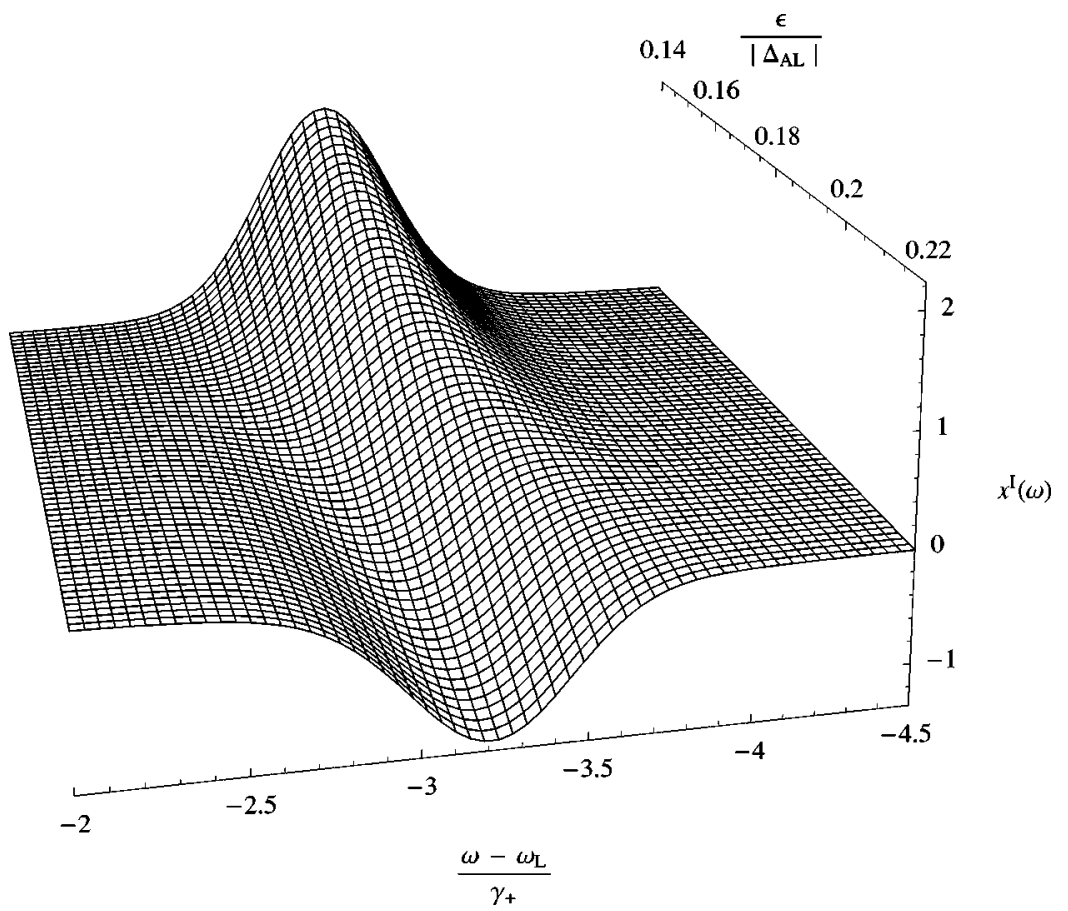

FIG. 18. The imaginary part of the linear susceptibility of the probe beam (absorption spectrum) as a function of the detuning of the probepump detuning frequency, $\omega-\omega_{L}$ and the driving field Rabi frequency, $\epsilon /\left|\Delta_{A L}\right|$, for $\gamma_{-} / \gamma_{+}=0.001$, $\gamma_{0} / \gamma_{+}=1, \epsilon /\left|\Delta_{A L}\right|=0.178$, and $\gamma_{p} / \gamma_{+}=0$. The inhomogeneous frequency distribution has a width $\Delta \omega_{I} /\left|\Delta_{A L}\right|=0.1$ and is centered below the laser field frequency, $\Delta_{A L}=\omega_{A}-\omega_{L}<0$. 


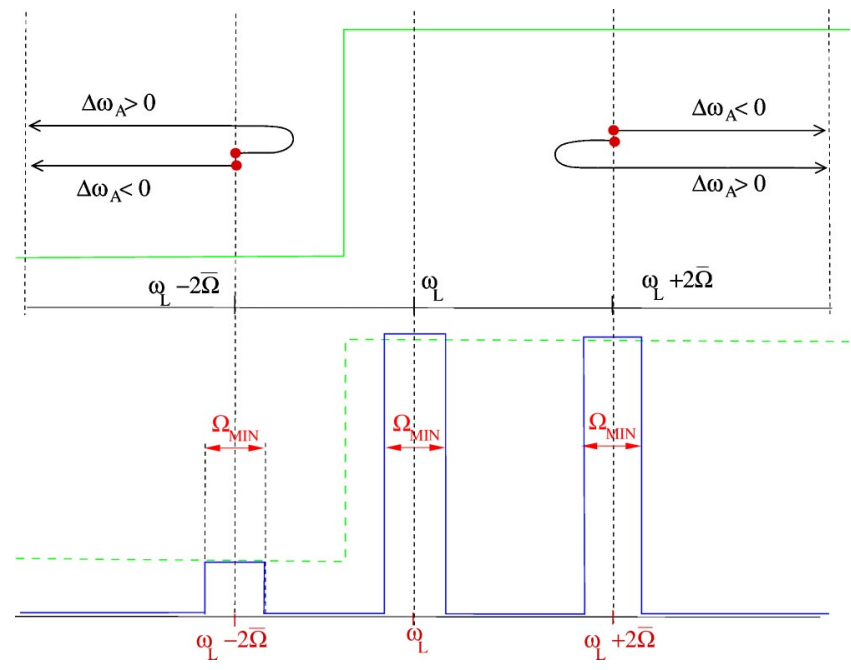

FIG. 19. The engineered photonic DOS proposed to quench the unfavorable members of the inhomogeneous quantum dot distribution. The upper panel shows the steplike density of states (the staircase curve) used in the absence of inhomogeneous broadening. The dot-arrow curves depict the evolution of the Mollow sideband peaks for a particular quantum dot as its deviation $\Delta \omega_{A}$ from the center of the inhomogeneous distribution is increased. The lower panel depicts a simple engineered photonic DOS, which quenches dots detuned by $\left|\Delta \omega_{A}\right|>\Omega_{M I N}$. This quenching prevents the smearing out of the switching effect.

propriate microcavity architecture within the 3D PBG material.

We present below a more detailed picture of our frequency selective atomic quenching mechanism. We denote the center of the inhomogeneous distribution by $\bar{\omega}_{A}$ and its detuning from the pump laser field frequency by $\bar{\Delta}_{A L} \equiv \bar{\omega}_{A}$ $-\omega_{L}$. We have

$$
\omega_{A}=\bar{\omega}_{A}+\Delta \omega_{A}
$$

$$
\begin{gathered}
\Delta_{A L}=\bar{\Delta}_{A L}+\Delta \omega_{A}, \\
\bar{\Omega} \equiv \sqrt{\epsilon^{2}+\frac{\bar{\Delta}_{2}^{A L}}{4}}, \\
\Omega=\sqrt{\epsilon^{2}+\frac{\Delta_{A L}^{2}}{4}}=\sqrt{\epsilon^{2}+\frac{\left(\bar{\Delta}_{A L}+\Delta \omega_{A}\right)^{2}}{4}} .
\end{gathered}
$$

Figure 20 presents the evolution of the Mollow peaks (thick dashed lines), and the atomic frequency (measured from the laser field frequency) for a specific atom as its detuning (from the center of the inhomogeneous distribution) increases (along the $y$ axis). The shaded vertical regions correspond to the engineered DOS presented in Fig. 19 (the left region corresponding to a decay constant $\gamma_{-}$, and the central and right regions corresponding to a decay constant $\gamma_{+}$). Starting from the center of the inhomogeneous distribution, $\omega_{A}=\bar{\omega}_{A}$, and increasing negative detuning $\left(\omega_{A}<\bar{\omega}_{A}\right)$, the left and right sidebands diverge from the central component according to Eq. (5.2a). For small positive detunings $\left(\omega_{A}>\bar{\omega}_{A}\right)$, the sidebands initially converge towards the central component, up to a minimum spectral distance equal to $\epsilon$, but then diverge for larger positive detunings. For a specific atom to contribute to the amplification process, its relevant Mollow components must lie in a region of nonzero density of states and thereby couple to pump and probe fields. This occurs in the horizontal shaded regions in Fig. 20. In the lower shaded region, the atomic detuning $\left(\Delta_{A L}\right)$ is negative, as required for switching. In the upper shaded region, the atomic detuning is positive $\left(\Delta_{A L}>0\right)$, and no switching occurs. These atoms only cause absorption of the probe beam and tend to offset the gain provided by the atoms in the lower shaded region. This cancellation effect can be alleviated by means of an incoherent pump that "bleaches" the positively detuned atoms in this upper region.

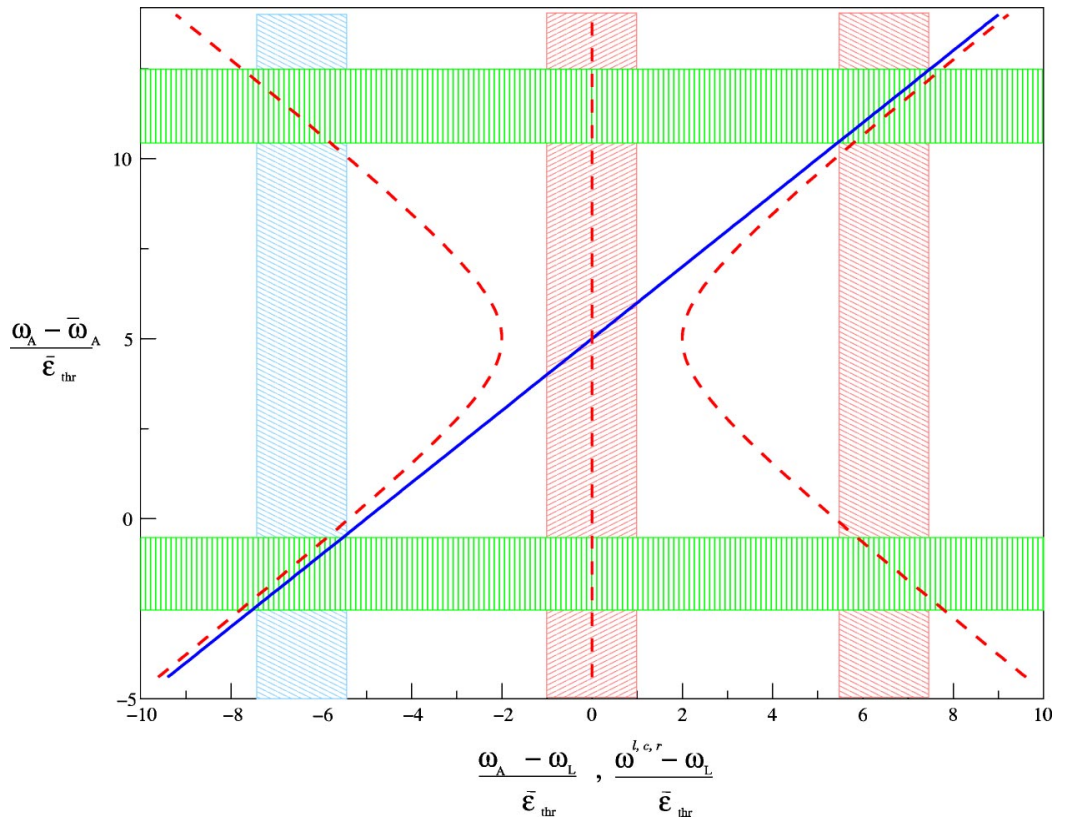

FIG. 20. The evolution of the atomic frequency detuning with respect to the laser field frequency (the thick continuous curve) and the spectral features (the left, central, and right components of the Mollow spectrum) (the thick dashed lines) as a function of the position of the atomic frequency with respect to the central frequency of the inhomogeneous distribution. To simplify the interpretation, the plot is rotated by $90^{\circ}$. The numerical values correspond to the parameters used above (with a negative detuning of the central frequency of the inhomogeneous broadening with respect to the laser field frequency). The driving field Rabi frequency is $\epsilon$ $=\epsilon_{t h r}$ and $\bar{\Delta}_{A L}=5 \epsilon_{t h r}$. 


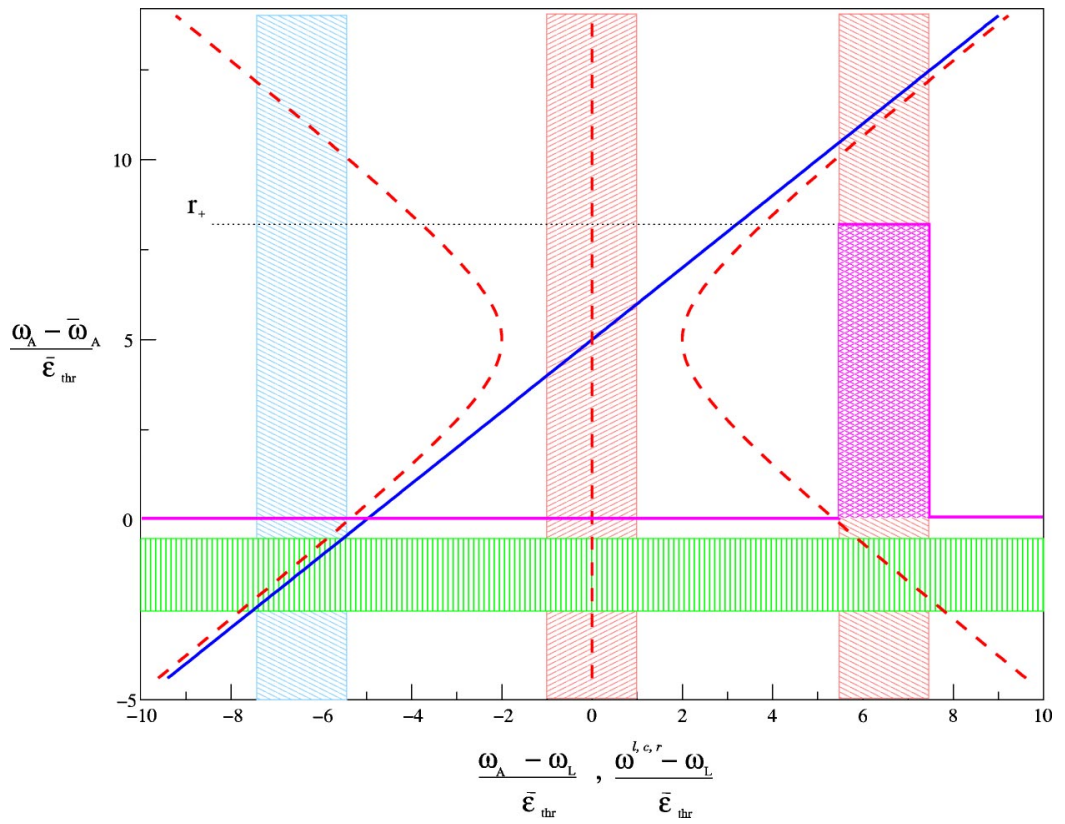

FIG. 21. Same as Fig. 20, but now we introduce a colored incoherent pump that saturates some members of the atomic distribution. The frequency profile of the pump rate is represented by the criss-crossed region (centered on the right sideband component of the Mollow spectrum), and its presence has as consequence the elimination of the upper permitted region in Fig. 20.

\section{Band gap engineering and incoherent pumping}

Incoherent pumping can be done using a third density of states band that bleaches the positively detuned atoms whose lower Mollow sideband (in the absence of bleaching) would contribute only to absorption within the probe spectrum. The pump profile needed is presented in Fig. 21. Detailed calculations show that the absorption of the probe field (Fig. 22) is almost identical to the one presented in Fig. 17, and the incoherent pump rate required is about $r_{+}=50 \gamma_{+}$.

\section{CONCLUSION}

We have analyzed the spectral response properties of twolevel atoms embedded in a photonic crystal and driven by an external laser field. This may have relevance to all-optical transistor action. As a function of the intensity of the pump (control) laser, the active region of a photonic material (the atomic system) switches from an absorptive medium (the atom spends most of its time in the ground state) to a gain medium (higher probability to find the atom in its excited state). The ability to invert a two-level atomic system in a photonic crystal, a phenomenon strictly forbidden in free space, is brought about by the additional degree of freedom present in the resonance fluorescence problem as a result of the structured radiation reservoir. The frequency dependence of the dressed-atom spectral features causes a "symmetry" breaking of the decay processes in the atomic relaxation cascade presented in Fig. 12. This is similar to the case of a

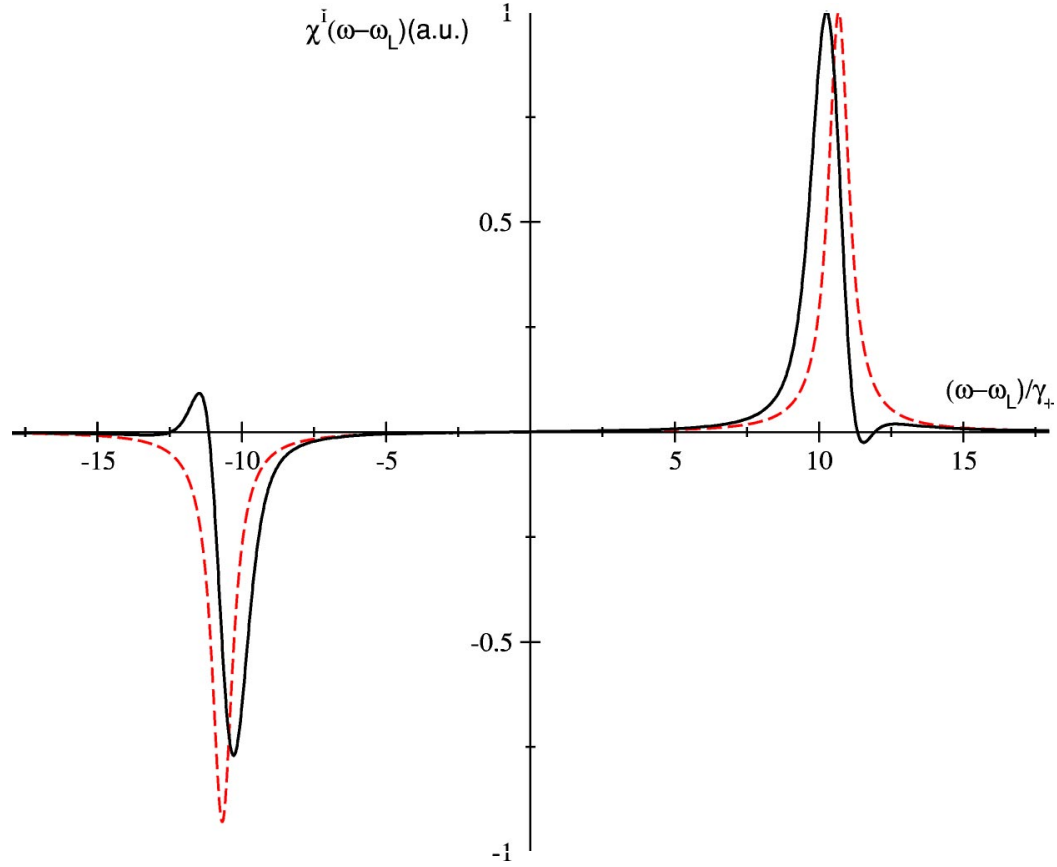

FIG. 22. The full recovery of the amplification effect in the presence of inhomogeneous broadening by band gap engineering and incoherent pump saturation mechanisms. The dashed curve represents the signal in the absence of inhomogeneous broadening. The continuous curve is obtained using the photonic DOS and the incoherent pump presented in Fig. 21. The width of the allowed passbands of the DOS is $\Delta \omega_{P B}$ $\approx \epsilon_{t h r}=0.183\left|\Delta_{A L}\right|$, and their magnitude is given by $\gamma_{-} / \gamma_{+}=0.001, \quad \gamma_{-} / \gamma_{+}=1$. The incoherent pump is characterized by $\Delta \omega_{P}=\Delta \omega_{P B} \approx \epsilon_{t h r}$, $\rho_{+} / \gamma_{+}=50, \rho_{-} / \gamma_{+}=0$. 
three-level atom in free space, where by introducing a specific structure in the atomic system (different decay rates for different relaxation channels), one breaks the "symmetry" of field assisted excitation and relaxation processes which characterizes a two-level atomic system, and makes possible the presence of positive atomic inversion [30].

We have investigated the spectral properties of the scattered radiation by the atomic system. We showed that in the presence of a colored vacuum, the sideband components of the scattered intensity can be strongly reduced and slightly narrowed. The dipolar dephasing influence on the scattered spectrum depends on the magnitude of the driving field intensity. For intensities below the switching threshold, the relative weight of the right sideband component is reduced, whereas for intensities above the threshold value, the dipolar dephasing has a more deleterious influence on the left sideband. We have also shown that a weak second probe laser beam experiences a substantial differential gain when the pump (control) laser intensity is near the switching threshold. This switching of the response of a probe beam (at a given frequency) to a small change in the pump (control) beam is absent in ordinary vacuum, and may offer a new approach to all-optical transistor action in photonic crystals. The switching power and the response time of an all-optical transistor can be dramatically decreased by including collective atomic effects (analyzed in Sec. III C) and non-Markovian effects [8]. We have shown that the correlations between the photons emitted by the atom are determined by the relative values of the photonic DOS at the dressed atomic frequencies. The degree of "antibunching" of the scattered photons can be controlled by the driving field intensity and may provide valuable information about the local environment encountered by the atom.

Collective atomic effects may further enhance the optical switching effect in photonic crystals. One enhancement is in the sharpness of the switching threshold. For $N$-atom switching, the change in the pump intensity required to pass through threshold scales linearly with $1 / N$. The spectral properties of the radiation emitted by the atomic ensemble also differ from those of a single atom. While in the immediate neighborhood of the threshold intensity the collective effects are manifest only in the $N^{2}$ scaling of the intensity spectrum (reminiscent of superradiance), above and below this critical region the gain spectrum widens (linearly with $N$ ). This correspondingly reduces the time scales for the response of the atomic system to external fields. We have shown that the sharpness of the switching threshold and the response time of an all-optical transistor can be dramatically reduced by including collective effects. This also provides a natural mechanism of broadening of the gain spectrum. As argued in the Introduction, such an all-optical device may have very low power requirements, with holding power in the nonowalt range and switching power of about 500 picowatt, and may achieve sub-picosecond switching times.

The application of the population inversion effect to an all-optical switch or transistor device will invariably require an active medium (ensemble of atoms or quantum dots), placed in a photonic crystal and interacting resonantly with coherent laser fields. Such ensembles exhibit a random spatial distribution of resonators, which experience different lo- cal environments or have different inherent resonance frequencies. We have analyzed the effects of inhomogeneous atomic line broadening on the all-optical switching effect. For small amounts of inhomogeneous broadening and away from the threshold intensity, we find that the amplifying spectral range simply becomes broader. For a large amount of inhomogeneous broadening, it is necessary to select a narrow spectral range around the central frequency of the distribution, in order to retain the amplification and the switching phenomena. This can be done through carefully engineered waveguide modes, which enable quenching of those parts of the atomic spectrum that degrade the amplification process. The development of appropriate photonic crystal architectures for "engineering the vacuum" in this manner may prove very rewarding for basic science and practical applications.

\section{APPENDIX: EVALUATION OF QUANTUM DEGREE OF SECOND-ORDER OPTICAL COHERENCE}

The evaluation of the second correlation function (3.17),

$$
G^{(2)}(\tau) \propto\left\langle\mathcal{E}^{(-)}(0) \mathcal{E}^{(-)}(\tau) \mathcal{E}^{(+)}(\tau) \mathcal{E}^{(+)}(0)\right\rangle_{s},
$$

follows an analogous path to the evaluation of the scattered spectrum. It proceeds by using the electric-field operators, Eq. (3.2), and the temporal dependence of the dressed-atomic operators found in Sec. II,

$$
\begin{gathered}
\left\langle R_{ \pm}(t)\right\rangle=\left\langle R_{ \pm}(0)\right\rangle e^{-\Gamma_{c o h} t}, \\
\left\langle R_{3}(t)\right\rangle=\left[\left\langle R_{3}(0)\right\rangle-\left\langle R_{3}\right\rangle^{s t}\right] e^{-\Gamma_{c o h} t}+\left\langle R_{3}\right\rangle^{s t},
\end{gathered}
$$

where $\Gamma_{p o p, c o h}$ are given by Eq. (2.16a). The explicit evaluation of Eq. (A1) involves 81 terms, but only 18 of them have a nonvanishing contribution (using the fact that the offdiagonal terms vanish in the long-time limit). We write the general result in the form

$$
G^{(2)}(\tau) \propto \sum_{k=1}^{18} T_{k}^{(4)},
$$

where $T_{k}^{(4)}$ is a generic four-atomic-operator average generated by the electric-field product appearing in Eq. (A1). For simplicity, we present here the evaluation of only four of such terms. For instance,

$$
\begin{gathered}
T_{1}^{(4)}=\gamma_{0}^{2} s^{4} c^{4}\left\langle R_{3}(0) R_{3}(t) R_{3}(t) R_{3}(0)\right\rangle_{s}, \\
T_{2}^{(4)}=\gamma_{0} \gamma_{+} s^{2} c^{6}\left\langle R_{3}(0) R_{+}(t) R_{-}(t) R_{3}(0)\right\rangle_{s}, \\
T_{4}^{(4)}=-\gamma_{0} \sqrt{\gamma_{-}} \sqrt{\gamma_{+}} s^{4} c^{4}\left\langle R_{3}(0) R_{3}(t) R_{-}(t) R_{+}(0)\right\rangle_{s} e^{-2 i \Omega t}, \\
T_{7}^{(4)}=\gamma_{0} \gamma_{+} s^{2} c^{6}\left\langle R_{3}(0) R_{+}(t) R_{3}(t) R_{-}(0)\right\rangle_{s} e^{+2 i \Omega t} .
\end{gathered}
$$

By collapsing the two-operator product to single operators, we obtain

$$
T_{1}^{(4)}=\gamma_{0}^{2} s^{4} c^{4}\left\langle R_{3}(0) R_{3}(0)\right\rangle_{s}
$$




$$
\begin{gathered}
T_{2}^{(4)}=\gamma_{0} \gamma_{+} s^{2} c^{6}\left\langle R_{3}(0) R_{22} R_{3}(0)\right\rangle_{s}, \\
T_{4}^{(4)}=\gamma_{0} \sqrt{\gamma_{-}} \sqrt{\gamma_{+}} s^{4} c^{4}\left\langle R_{3}(0) R_{-}(t) R_{+}(0)\right\rangle_{s} e^{-2 i \Omega t}, \\
T_{7}^{(4)}=-\gamma_{0} \gamma_{+} s^{2} c^{6}\left\langle R_{3}(0) R_{+}(t) R_{-}(0)\right\rangle_{s} e^{+2 i \Omega t}
\end{gathered}
$$

The temporal evolution of the four-operator product in Eqs. (A5) is then found using the quantum regression theorem, and (after calculating the steady-state averages, \langle\rangle$_{s}$ ), we obtain

$$
\begin{gathered}
T_{1}^{(4)}=\gamma_{0}^{2} s^{4} c^{4}, \\
T_{2}^{(4)}=\gamma_{0} \gamma_{+} s^{2} c^{6} \pi_{1}, \\
T_{4}^{(4)}=\gamma_{0} \sqrt{\gamma_{-}} \sqrt{\gamma_{+}} s^{4} c^{4} \pi_{1} e^{-\Gamma_{c o h} t} e^{-2 i \Omega t}, \\
T_{7}^{(4)}=-\gamma_{0} \gamma_{+} s^{2} c^{6} \pi_{1} e^{-\Gamma_{c o h} t} e^{+2 i \Omega t} .
\end{gathered}
$$

Here, $\pi_{1}, \pi_{2}$ are the steady-state dressed-atomic populations for the ground and excited states, respectively. The rest of 14 terms in Eq. (A3) are evaluated in a similar fashion, and we obtain

$$
\begin{aligned}
G^{(2)}(\tau) \propto & \left(\gamma_{0}^{2}+\gamma_{-} \gamma_{+}\right) s^{4} c^{4}+2 \gamma_{0} s^{2} c^{2}\left(\gamma_{+} c^{4} \pi_{2}+\gamma_{-} s^{4} \pi_{1}\right) \\
& -2 \gamma_{0} s^{2} c^{2}\left(\sqrt{\gamma_{-}} \sqrt{\gamma_{+}} s^{2} c^{2}+\gamma_{+} c^{4} \pi_{2}\right. \\
& \left.+\gamma_{-} s^{4} \pi_{1}\right) e^{-\Gamma_{c o h}} \cos (2 \Omega t), \\
\lim _{\tau \rightarrow \infty} G^{(2)}(\tau) \propto & \left(\gamma_{0}^{2}+\gamma_{-} \gamma_{+}\right) s^{4} c^{4}+2 \gamma_{0} s^{2} c^{2}\left(\gamma_{+} c^{4} \pi_{2}+\gamma_{-} s^{4} \pi_{1}\right),
\end{aligned}
$$

such that the degree of second-order coherence function becomes

$$
\begin{aligned}
g^{(2)}(\tau)= & 1-2 \gamma_{0} e^{-\Gamma_{c o h} \tau} \cos (2 \Omega \tau) \\
& \times \frac{\sqrt{\gamma_{-}} \sqrt{\gamma_{+}} c^{2} s^{2}+\gamma_{+} c^{4} \pi_{2}+\gamma_{-} s^{4} \pi_{1}}{\left(\gamma_{0}^{2}+\gamma_{-} \gamma_{+}\right) c^{2} s^{2}+2 \gamma_{0}\left(\gamma_{+} c^{4} \pi_{2}+\gamma_{-} s^{4} \pi_{1}\right)} .
\end{aligned}
$$

[1] S. John, Phys. Rev. Lett. 53, 2169 (1984).

[2] K. M. Ho, C. T. Chan, and C. M. Soukoulis, Phys. Rev. Lett. 65, 3152 (1990).

[3] E. Yablonovitch, T. J. Gmitter, and K. M. Leung, Phys. Rev. Lett. 67, 2295 (1991).

[4] E. Yablonovitch, Phys. Rev. Lett. 58, 2059 (1987).

[5] S. John, Phys. Rev. Lett. 58, 2486 (1987).

[6] S. John and T. Quang, Phys. Rev. A 50, 1764 (1994); A. Kaufman, G. Kurizki, and B. Sherman, J. Mod. Opt. 41, 353 (1994); M. Lewenstein, J. Zakrzewski, and T. W. Mossberg, Phys. Rev. A 38, 808 (1988); Y. Yang and S. Y. Zhu, ibid. 62, 013805 (2000).

[7] S. John and T. Quang, Phys. Rev. Lett. 78, 1888 (1997).

[8] M. Florescu and S. John, Phys. Rev. A 64, 033801 (2001).

[9] S. John and M. Florescu, J. Opt. A, Pure Appl. Opt. 3, S103 (2001).

[10] H. M. Gibbs, Optical Bistability: Controlling Light with Light (Academic Press, New York, 1985).

[11] A. Chutinan, S. John, and O. Toader, Phys. Rev. Lett. 90, 123901 (2003).

[12] R. Wang and S. John (unpublished).

[13] O. Toader and S. John, Science 229, 5519 (2001).

[14] O. Painter et al., Science 284, 1819 (1999).

[15] O. Toader, S. John, and K. Busch, Opt. Express 8, 272 (2001).

[16] H. Carmichael, An Open Systems Approach to Quantum Optics
(Springer-Verlag, Berlin, 1993).

[17] M. Florescu, Ph.D. thesis, University of Toronto, 2003 (unpublished).

[18] C. Cohen-Tannoudji, J. Dupont-Roc, and S. Grynberg, AtomPhoton Interactions (Wiley-Interscience, New York, 1992); H. Freedhoff and T. Quang, J. Opt. Soc. Am. B 12, 474 (1993).

[19] M. Florescu, S. John, and V. Rupasov (unpublished).

[20] T. W. Mosseberg and M. Lewenstein, J. Opt. Soc. Am. B 10, 340 (1993).

[21] H. Freedhofff and T. Quang, J. Opt. Soc. Am. B 12, 9 (1995).

[22] M. Lax, Phys. Rev. 172, 350 (1968).

[23] R. Hanbury-Brown and R. Q. Twiss, Nature (London) 177, 27 (1956).

[24] G. Compagno and F. Persico, Phys. Rev. A 25, 3138 (1982).

[25] A. Aspect et al., Phys. Rev. Lett. 45, 617 (1980).

[26] B. R. Mollow, Phys. Rev. A 5, 2217 (1972).

[27] H. Freedhofff and T. Quang, Phys. Rev. Lett. 72, 474 (1994).

[28] C. Cohen Tannoudji and S. Reynaud, in Proceedings of an International Conference at the University of Rochester, 1977, edited by J. H. Eberly and P. Lambropoulos (Wiley, New York, 1978).

[29] P. R. Hemmer et al., Phys. Rev. Lett. 77, 1468 (1996).

[30] H. Lee, Y. Rostovtsev, and M. O. Scully, Phys. Rev. A 62, 063804 (2000). 